\title{
Effects of Non-uniform Temperature Gradients on Triple Diffusive Surface Tension Driven Magneto Convection in a Composite Layer
}

\author{
MANJUNATHA $\mathbf{N}^{1, *}$, SUMITHRA $\mathbf{R}^{2}$ \\ ${ }^{1}$ School of Applied Sciences, REVA University, Bengaluru-560064, India \\ 2 Department of Mathematics, Government Science College, Bengaluru-560001, India \\ *Corresponding Author: manjunatha.n@ reva.edu.in
}

Received October 08, 2019; Revised November 20, 2019; Accepted November 27, 2019

Copyright $@ 2019$ by authors, all rights reserved. Authors agree that this article remains permanently open access under the terms of the Creative Commons Attribution License 4.0 International License

\begin{abstract}
The problem of triple diffusive surface tension driven magneto convection in a composite layer is investigated for linear, parabolic and inverted parabolic temperature profiles. The corresponding thermal Marangoni numbers are obtained analytically depending on various physical parameters of interest. The parameters which are effective to enhance convection and to control convection are determined. From the investigation it is found that the linear profile is suitable for fluid layer dominant composite layer where as parabolic and inverted parabolic profiles are conducive for the porous layer dominant composite layer.
\end{abstract}

Keywords Triple diffusive, Magneto convection, Surface tension, temperature profiles, Composite layer.

\section{Introduction}

There are many fluid systems containing more than two components occurring in nature. The subject of systems having multi components in porous and fluid layers has attracted many researchers due to its importance in the study of crystal growth, geothermally heated lakes, earth core, solidification of molten alloys, underground water flow, acid rain effects, natural phenomena such as contaminant transport, warming of stratosphere, magmas and sea water etc. In many situations particularly in geophysics, astrophysics and in some industrial problems maintaining a uniform temperature gradient is a limitation and non-uniform temperature/concentration gradients is a reality. In that case, the stability or instability of a fluid in the presence of a nonlinear temperature profile is of practical importance and has not been given much attention.

For the single fluid layer, Melviana Johnson Fu et al. [1] have investigated the effect of a non-uniform basic temperature gradient and magnetic field on the onset of Marangoni convection in a horizontal micro polar fluid layer using the Rayleigh-
Ritz technique. They found that the micro polar fluid layer heated from below is more stable compared to the classical fluid layer. Using linear stability analysis, Isa et al. [2, 3, 4] have explored the impact of non-uniform temperature gradient and magnetic field on Marangoni and Benard-Marangoni convection in a horizontal fluid layer heated from below and cooled from above. Norihan Md. Arifin et al. [5] have studied the effect of a non-uniform basic temperature gradient and magnetic field on the onset of Benard-Marangoni convection in a horizontal micro polar fluid layer using Rayleigh-Ritz technique. The combined effects of vertical magnetic field and non-uniform temperature profiles on the onset of steady Marangoni convection in a horizontal layer of micro polar fluid are investigated by Mahmud et al. [6]. The effects of electric field and non-uniform basic temperature gradient on the onset of Rayleigh-Benard convection in a micro polar fluid are studied by Pranesh and Riya Baby [7] using the Galerkin technique. The onset of convection is discussed for six nonlinear temperature profiles. Pranesh and Kiran [8] have studied the effect of non-uniform temperature gradient on the onset of Rayleigh-Benard magneto convection in a micropolar fluid with Maxwell-Cattaneo law using the Galerkin technique. The onset of convection is discussed for six non-linear temperature profiles. Thadathil Varghese Joseph et al. [9] studied effect of non-uniform basic temperature gradient on the onset of Rayleigh-Benard-Marangoni electro-convection in a micropolar fluid. Recently, Azmi and Idris [10] have applied the linear stability analysis to study the effects of non-uniform basic temperature gradients on the onset of Rayleigh-BenardMarangoni magneto convection in a micro polar fluid by using the Galerkin technique. They considered three temperature profiles and their comparative influence on onset of convection is discussed. Using linear stability analysis, Sandhya and Sangeetha George [11] have studied the different temperature gradients and rotation on the onset of Marangoni convection in a fluid which has suspended particles in it and confined 
between an upper free, adiabatic and lower rigid, isothermal boundaries under micro gravity condition.

The literature cited above is concerned with convection in the presence of a non-uniform temperature gradient in the fluid layer. However, many engineering problems involve porous media and in such cases it is essential to control convection. This can be achieved by using a non-uniform temperature gradient in the porous layer. In view of this, Shivakumara [12] has studied the onset of convection in a layer of couple-stress fluidsaturated porous medium is investigated for different types of basic temperature gradients using the Galerkin technique. It is observed that the critical thermal depth decreases marginally with an increase in the couple-stress parameter. Shivakumara et al. [13] studied the simultaneous effect of local thermal non equilibrium, vertical heterogeneity of permeability and nonuniform basic temperature gradient on the criterion for the onset of Darcy-Benard convection. The eigenvalue problem is solved numerically using the Galerkin method. Shivakumara et al. [14] have investigated the effect of six different temperature gradients on the onset of convection in a couple stress fluid saturated porous medium using the Galerkin technique. They found that parabolic and inverted parabolic basic temperature profiles have the same effect on the onset of convection.

For the composite layers, Manjunatha and Sumithra [15, $16,17]$ are investigated the combined effects of magnetic field and non-uniform basic temperature gradients on two and three component convection in two layer system.

In this paper, the lower rigid surface of the porous layer and the upper free surface are considered to be insulating to temperature, insulating to both solute concentration perturbations. At the upper free surface, the surface tension effects depending on temperature and salinities are considered. At the interface, the normal and tangential components of velocity, heat and heat flux, mass and mass flux are assumed to be continuous. The resulting eigenvalue problem is solved exactly for linear, parabolic and inverted parabolic temperature profiles and analytical expressions of the thermal Marangoni number are obtained. Effects of variation of different physical parameters on the thermal Marangoni numbers for the profiles are compared.

\section{Mathematical formulation}

We consider a three different diffusing components with different molecular diffusivities, electrically conducting fluid layer of thickness $d$ horizontally isotropic sparsely packed porous layer of saturated with same fluid of thickness $d_{m}$ in the presence of magnetic field $H_{0}$ in the vertical $z$ - direction. The lower surface of the porous layer is considered to be rigid and the upper surface of the fluid layer is free at which the surface tension effects depending on temperature and both the species concentrations is considered. Both the boundaries are kept at different constant temperatures and salinities. A Cartesian coordinate system is chosen with the origin at the interface between porous and fluid layers and the $z$ - axis, vertically upwards. The basic equations for fluid and porous layer respec-

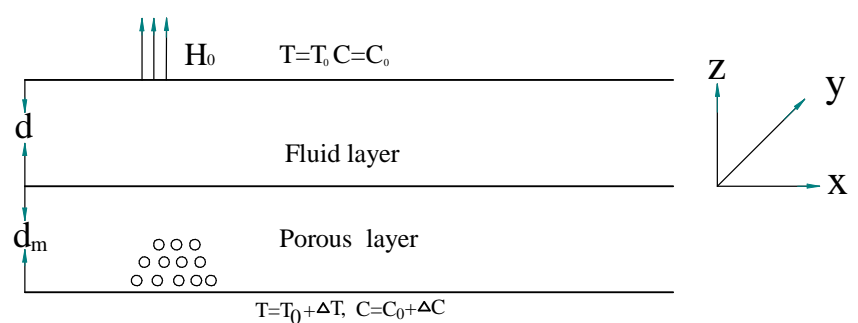

Figure 1. Physical configuration

tively as,

$$
\begin{aligned}
& \nabla \cdot \vec{q}=0 \\
& \nabla \cdot \vec{H}=0 \\
& \rho_{0}\left[\frac{\partial \vec{q}}{\partial t}+(\vec{q} \cdot \nabla)\right]=-\nabla P+\mu \nabla^{2} \vec{q} \\
& \frac{\partial T}{\partial t}+(\vec{q} \cdot \nabla) T=\kappa \nabla^{2} T \\
& \frac{\partial C_{1}}{\partial t}+(\vec{q} \cdot \nabla) C_{1}=\kappa_{1} \nabla^{2} C_{1} \\
& \frac{\partial C_{2}}{\partial t}+(\vec{q} \cdot \nabla) C_{2}=\kappa_{2} \nabla^{2} C_{2} \\
& \frac{\partial \vec{H}}{\partial t}=\nabla \times \vec{q} \times \vec{H}+\nu_{m} \nabla^{2} \vec{H} \\
& \nabla_{m} \cdot \overrightarrow{q_{m}}=0 \\
& \nabla_{m} \cdot \overrightarrow{H_{m}}=0 \\
& \rho_{0}\left[\frac{1}{\varepsilon} \frac{\partial \overrightarrow{q_{m}}}{\partial t}+\frac{1}{\varepsilon^{2}}\left(\overrightarrow{q_{m}} \cdot \nabla_{m}\right) \overrightarrow{q_{m}}\right]=-\nabla_{m} P_{m} \\
& +\mu_{m} \nabla_{m}^{2} \overrightarrow{q_{m}}-\frac{\mu}{K} \overrightarrow{q_{m}} \\
& A \frac{\partial T_{m}}{\partial t}+\left(\overrightarrow{q_{m}} \cdot \nabla_{m}\right) T_{m}=\kappa_{m} \nabla_{m}^{2} T_{m} \\
& \varepsilon \frac{\partial C_{m 1}}{\partial t}+\left(\overrightarrow{q_{m}} \cdot \nabla_{m}\right) C_{m 1}=\kappa_{m 1} \nabla_{m}^{2} C_{m 1} \\
& \varepsilon \frac{\partial C_{m 2}}{\partial t}+\left(\overrightarrow{q_{m}} \cdot \nabla_{m}\right) C_{m 2}=\kappa_{m 2} \nabla_{m}^{2} C_{m 2} \\
& \frac{\partial \overrightarrow{H_{m}}}{\partial t}=\nabla_{m} \times \overrightarrow{q_{m}} \times \overrightarrow{H_{m}}+\nu_{e m} \nabla_{m}^{2} \overrightarrow{H_{m}}
\end{aligned}
$$

Here $\vec{q}=(u, v, w)$ is the velocity vector, $\vec{H}$ is the magnetic field, $\rho_{0}$ is the fluid density, $t$ is the time, $\mu$ is the fluid viscosity, $P=p+\frac{\mu_{p} H^{2}}{2}$ is the total pressure, $T$ is the temperature, $\kappa$ is the thermal diffusivity of the fluid, $\kappa_{1}$ and $\kappa_{2}$ are the solute 1 and solute 2 diffusivity of the fluid in the fluid layer, $C_{1}$ and $C_{2}$ are the concentration 1 and concentration 2 for the fluid in the fluid layer, $\nu_{m}=\frac{1}{\mu_{p} \sigma}$ is the magnetic viscosity, $K$ is the permeability of the porous medium, $A=\frac{\left(\rho_{0} C_{p}\right)_{m}}{\left(\rho C_{p}\right)_{f}}$ is the ratio of heat capacities, $C_{p}$ is the specific heat, $\varepsilon$ is the porosity, $\mu_{m}$ is the effective viscosity of the fluid in the porous layer, $\kappa_{m 1}$ and $\kappa_{m 2}$ is the solute 1 and solute 2 diffusivity of the fluid in porous layer, $C_{m 1}$ and $C_{m 2}$ are the concentration1 and concentration2 for the fluid in porous layer, $\nu_{e m}=\frac{\nu_{m}}{\varepsilon}$ is the effective magnetic viscosity and the subscripts ' $m$ ' and 'f' refer to the porous medium and the fluid respectively.

The equations (1) to (14) have a basic steady solution for 
fluid and porous layer respectively and they are

$$
\begin{array}{r}
\left\{\vec{q}=\overrightarrow{q_{b}}, P=P_{b}(z), T=T_{b}(z), C_{1}=C_{1 b}(z),\right. \\
\left.C_{2}=C_{2 b}(z), \vec{H}=H_{0}(z)\right\} \\
\left\{\overrightarrow{q_{m}}=\overrightarrow{q_{m b}}, P_{m}=P_{m b}\left(z_{m}\right), T_{m}=T_{m b}\left(z_{m}\right),\right. \\
\left.C_{1 m}=C_{1 m b}\left(z_{m}\right), C_{2 m}=C_{2 m b}\left(z_{m}\right)\right\} \\
-\frac{\partial T_{b}}{\partial z}=\frac{\left(T_{0}-T_{u}\right)}{d} h(z) \quad 0 \leq z \leq d \\
-\frac{\partial T_{m b}}{\partial z_{m}}=\frac{\left(T_{l}-T_{0}\right)}{d_{m}} h_{m}\left(z_{m}\right)-d_{m} \leq z_{m} \leq 0 \\
C_{1 b}(z)=C_{10}-\frac{\left(C_{10}-C_{1 u}\right) z}{d} \quad 0 \leq z \leq d \\
C_{1 m b}\left(z_{m}\right)=C_{10}-\frac{\left(C_{1 l}-C_{10}\right) z_{m}}{d_{m}}-d_{m} \leq z_{m} \leq 0 \\
C_{2 b}(z)=C_{20}-\frac{\left(C_{20}-C_{2 u}\right) z}{d} \quad 0 \leq z \leq d \\
C_{2 m b}\left(z_{m}\right)=C_{20}-\frac{\left(C_{2 l}-C_{20}\right) z_{m}}{d_{m}}-d_{m} \leq z_{m} \leq 0
\end{array}
$$

\section{Where}

$h(z)$ and $h_{m}\left(z_{m}\right)$ are temperature gradients in fluid and porous layers respectively and the subscript ' $b$ ' denotes the basic state. The interface temperature is $T_{0}=$ $\frac{\kappa d_{m} T_{u}+\kappa_{m} d T_{l}}{\kappa d_{m}+\kappa_{m} d}$ and the interface concentrations are $C_{10}=$ $\frac{\kappa_{1} d_{m} C_{1 u}+\kappa_{1 m} d C_{1 l}}{\kappa_{1} d_{m}+\kappa_{1 m} d}, C_{20}=\frac{\kappa_{2} d_{m} C_{2 u}+\kappa_{2 m} d C_{2 l}}{\kappa_{2} d_{m}+\kappa_{2 m} d}$.

To examine the stability of the system, we give a small perturbation to the system as

$$
\begin{array}{r}
\left\{\vec{q}=\overrightarrow{q_{b}}+\vec{q}^{\prime}, P=P_{b}+P^{\prime}, T=T_{b}(z)+\theta,\right. \\
C_{1}=C_{1 b}(z)+S_{1}, C_{2}=C_{2 b}(z)+S_{2}, \\
\left.\vec{H}=H_{0}(z)+\overrightarrow{H^{\prime}}\right\} \\
\left\{\overrightarrow{q_{m}}=\overrightarrow{q_{m b}}+\overrightarrow{q_{m}}, P_{m}=P_{m b}+P_{m}^{\prime},\right. \\
T_{m}=T_{m b}\left(z_{m}\right)+\theta_{m}, \\
C_{1 m}=C_{1 m b}\left(z_{m}\right)+S_{m 1}, \\
C_{2 m}=C_{2 m b}\left(z_{m}\right)+S_{m 2}, \\
\left.\overrightarrow{H_{m}}=H_{0}\left(z_{m}\right)+\overrightarrow{H^{\prime}}\right\}
\end{array}
$$

Where the primed quantities are the dimensionless ones. Equations (23) \& (24) are substituted into the (1) to (14), apply curl twice to eliminate the pressure term from (3) \& (10) and then variables are nondimensionalised for fluid and porous layer.

To render the equations nondimensional, we choose different scales for the two layers ( Chen and Chen [18], Nield [19]), so that both layers are of unit length such that $(x, y, z)=d\left(x^{\prime}, y^{\prime}, z^{\prime}\right),\left(x_{m}, y_{m}, z_{m}\right)=d_{m}\left(x_{m}^{\prime}, y_{m}^{\prime}, z_{m}^{\prime}-1\right)$. and $0 \leq z_{m} \leq 1$ respectively

$$
\begin{array}{r}
\frac{1}{P r} \frac{\partial\left(\nabla^{2} W\right)}{\partial t}=\nabla^{4} W+Q \tau_{f m} \frac{\partial\left(\nabla^{2} H_{z}\right)}{\partial z} \\
\frac{\partial \theta}{\partial t}=W h(z)+\nabla^{2} \theta \\
\frac{\partial S_{1}}{\partial t}=W+\tau_{1} \nabla^{2} S_{1} \\
\frac{\partial S_{2}}{\partial t}=W+\tau_{2} \nabla^{2} S_{2} \\
\frac{\partial H_{z}}{\partial t}=\frac{\partial W}{\partial t}+\tau_{f m} \nabla^{2} H_{z} \\
\frac{\beta^{2}}{P r_{m}} \frac{\partial\left(\nabla_{m}^{2} W_{m}\right)}{\partial t}=\widehat{\mu} \beta^{2} \nabla_{m}^{4} W_{m}-\nabla_{m}^{2} W_{m} \\
+Q_{m} \tau_{m m} \beta^{2} \frac{\partial\left(\nabla_{m}^{2} H_{z m}\right)}{\partial z_{m}} \\
A \frac{\partial \theta_{m}}{\partial t}=W_{m} h_{m}\left(z_{m}\right)+\nabla_{m}^{2} \theta_{m} \\
\varepsilon \frac{\partial S_{m 1}}{\partial t}=W_{m}+\tau_{m 1} \nabla_{m}^{2} S_{m 1} \\
\varepsilon \frac{\partial S_{m 2}}{\partial t}=W_{m}+\tau_{m 2} \nabla_{m}^{2} S_{m 2} \\
\varepsilon \frac{\partial H_{z m}}{\partial t}=\frac{\partial W_{m}}{\partial t}+\tau_{m m} \nabla^{2} H_{z m}
\end{array}
$$

Here, for the fluid layer $\operatorname{Pr}=\frac{\nu}{\kappa}$ is the Prandtl number, $Q=\frac{\mu_{p} H_{0}^{2} d^{2}}{\mu \kappa \tau_{f m}}$ is the Chandrasekhar number, $\tau_{f m}=\frac{\nu_{m v}}{\kappa}$ is the diffusivity ratio for fluid layer, $\tau_{1}=\frac{\kappa_{1}}{\kappa}$ and $\tau_{2}=\frac{\kappa_{2}}{\kappa}$ are the ratios of solute1 and solute 2 diffusivity to thermal diffusivity fluid in fluid layer. For the porous layer, $P r_{m}=\frac{\varepsilon \nu_{m}}{\kappa_{m}}$ is the Prandtl number, $Q_{m}=\frac{\mu_{p} H_{0}^{2} d_{m}^{2}}{\mu \kappa_{m} \tau_{m m}}=Q \varepsilon \widehat{d^{2}}$ is the Chandrasekhar number, $\tau_{m m}=\frac{\nu_{e m}}{\kappa_{m}}$ diffusivity ratio in the porous layer, $\beta=\sqrt{\frac{K}{d_{m}^{2}}}$ is the porous parameter, $\widehat{\mu}=\frac{\mu_{m}}{\mu}$ is viscosity ratio, $\tau_{m 1}=\frac{\kappa_{m 1}}{\kappa_{m}}$ and $\tau_{m 2}=\frac{\kappa_{m 2}}{\kappa_{m}}$ are the ratios of solute 1 and solute 2 diffusivity to thermal diffusivity of the porous layer, $h(z)$ and $h_{m}\left(z_{m}\right)$ are the non-dimensional temperature gradients with $\int_{0}^{1} h(z) d z=1$ and $\int_{0}^{1} h_{m}\left(z_{m}\right) d z_{m}=1, \theta$ and $\theta_{m}$ are the temperature in fluid and porous layers respectively, $S_{1}, S_{2}$ and $S_{m 1}, S_{m 2}$ are the concentrations in fluid and porous layer respectively and $W$ and $W_{m}$ are the dimensionless vertical velocity distributions in fluid and porous layer respectively.

Omitting the primes for simplicity, we get in $0 \leq z \leq 1$ We apply normal mode expansion on dependent variables as 
follows,

$$
\begin{gathered}
{\left[\begin{array}{c}
W \\
\theta \\
S_{1} \\
S_{2} \\
H
\end{array}\right]=\left[\begin{array}{c}
W(z) \\
\theta(z) \\
S_{1}(z) \\
S_{2}(z) \\
H(z)
\end{array}\right] f(x, y) e^{n t}} \\
{\left[\begin{array}{c}
W_{m} \\
\theta_{m} \\
S_{m 1} \\
S_{m 2} \\
H_{m}
\end{array}\right]=\left[\begin{array}{c}
W_{m}\left(z_{m}\right) \\
\theta_{m}\left(z_{m}\right) \\
S_{m 1}\left(z_{m}\right) \\
S_{m 2}\left(z_{m}\right) \\
H_{m}\left(z_{m}\right)
\end{array}\right] f_{m}\left(x_{m}, y_{m}\right) e^{n_{m} t}}
\end{gathered}
$$

with $\nabla_{2}^{2} f+a^{2} f=0$ and $\nabla_{2 m}^{2} f_{m}+a_{m}^{2} f_{m}=0$. Here $a$ and $a_{m}$ are the nondimensional horizontal wave numbers, $n$ and $n_{m}$ are the frequencies. Since the dimensional horizontal wave numbers must be the same for the fluid and porous layers, we must have $\frac{a}{d}=\frac{a_{m}}{d_{m}}$ and hence $a_{m}=\widehat{d a}$.

Introducing Eqs. (35) and (36) into the Eqs. (25) to (34) then we get an Eigen value problem consisting of the following differential equation in $0 \leq z \leq 1$ and $0 \leq z_{m} \leq 1$ respectively

$$
\begin{array}{r}
\left(D^{2}-a^{2}+\frac{n}{P r}\right)\left(D^{2}-a^{2}\right) W=-Q \tau_{f m} D\left(D^{2}-a^{2}\right) H \\
\left(D^{2}-a^{2}+n\right) \theta+W h(z)=0 \\
\left(\tau_{1}\left(D^{2}-a^{2}\right)+n\right) S_{1}+W=0 \\
\left(\tau_{2}\left(D^{2}-a^{2}\right)+n\right) S_{2}+W=0 \\
\tau_{f m}\left(D^{2}-a^{2}+n\right) H+D W=0 \\
{\left[\left(D_{m}^{2}-a_{m}^{2}\right) \widehat{\mu} \beta^{2}+\frac{n_{m} \beta^{2}}{P r_{m}}-1\right]\left(D_{m}^{2}-a_{m}^{2}\right) W_{m}} \\
=-Q_{m} \tau_{m m} \beta^{2} D_{m}\left(D_{m}^{2}-a_{m}^{2}\right) H_{m} \\
\left(D_{m}^{2}-a_{m}^{2}+A n_{m}\right) \theta_{m}+W_{m} h_{m}\left(z_{m}\right)=0 \\
\left(\tau_{m 1}\left(D_{m}^{2}-a_{m}^{2}\right)+n_{m} \varepsilon\right) S_{m 1}+W_{m}=0 \\
\left(\tau_{m 2}\left(D_{m}^{2}-a_{m}^{2}\right)+n_{m} \varepsilon\right) S_{m 2}+W_{m}=0 \\
\tau_{m m}\left(D_{m}^{2}-a_{m}^{2}+n_{m} \varepsilon\right) H_{m}+D_{m} W_{m}=0
\end{array}
$$

Assume that the principle of exchange of instabilities holds for present problem, hence we take $n=n_{m}=0$. Eliminating the magnetic field in Eqs. (41) and (46). The Eigen value problem becomes, in $0 \leq z \leq 1$ and $0 \leq z_{m} \leq 1$ respectively

$$
\begin{array}{r}
\left(D^{2}-a^{2}\right)^{2} W=Q D^{2} W(47) \\
\left(D^{2}-a^{2}\right) \theta+W h(z)=0(48) \\
\tau_{1}\left(D^{2}-a^{2}\right) S_{1}+W=0(49) \\
\tau_{2}\left(D^{2}-a^{2}\right) S_{2}+W=0(50) \\
{\left[\left(D_{m}^{2}-a_{m}^{2}\right) \widehat{\mu} \beta^{2}-1\right]\left(D_{m}^{2}-a_{m}^{2}\right) W_{m}=Q_{m} \beta^{2} D_{m}^{2} W_{m}(51)} \\
\left(D_{m}^{2}-a_{m}^{2}\right) \theta_{m}+W_{m} h_{m}\left(z_{m}\right)=0(52) \\
\tau_{m 1}\left(D_{m}^{2}-a_{m}^{2}\right) S_{m 1}+W_{m}=0(53) \\
\tau_{m 2}\left(D_{m}^{2}-a_{m}^{2}\right) S_{m 2}+W_{m}=0(54)
\end{array}
$$

\section{Boundary conditions}

The boundary conditions are nondimensionalised then subjected to normal mode analysis and finally they take the form

$$
\begin{array}{r}
{\left[D^{2} W(1)+M a^{2} \theta(1)+M_{s 1} a^{2} S_{1}(1)\right.} \\
\left.+M_{s 2} a^{2} S_{2}(1)\right]=0, \\
W(1)=D \theta(1)=D S_{1}(1)=D S_{2}(1)=0, \\
W_{m}(0)=D_{m} W_{m}(0)=D_{m} \theta_{m}(0)=0, \\
D_{m} S_{m 1}(0)=D_{m} S_{m 2}(0)=0, \\
\widehat{T} W(0)=W_{m}(1), \widehat{T} \widehat{d} D W(0)=D_{m} W_{m}(1), \\
\widehat{T} \widehat{d^{2}}\left(D^{2}+a^{2}\right) W(0)=\widehat{\mu}\left(D_{m}^{2}+a_{m}^{2}\right) W_{m}(1), \\
\widehat{T} \widehat{d^{3}} \beta^{2}\left(D^{3} W(0)-3 a^{2} D W(0)\right)=D_{m} W_{m}(1) \\
+\widehat{\mu} \beta^{2}\left(D_{m}^{3} W_{m}(1)-3 a_{m}^{2} D_{m} W_{m}(1)\right), \\
\theta(0)=\widehat{T} \theta_{m}(1), D \theta(0)=D_{m} \theta_{m}(1), \\
S_{1}(0)=\widehat{S_{1}} S_{m 1}(1), D S_{1}(0)=D_{m} S_{m 1}(1), \\
S_{2}(0)=\widehat{S_{2}} S_{m 2}(1), D S_{2}(0)=D_{m} S_{m 2}(1)
\end{array}
$$

Where

$\widehat{S_{1}}=\frac{\kappa_{s 1}}{\kappa_{s 1 m}}, \widehat{S_{2}}=\frac{\kappa_{s 2}}{\kappa_{s 2 m}}$ are the ratios of solute1 and solute2 diffusivities of fluid layer to those of porous layer respectively, $\widehat{d}=\frac{d_{m}}{d}$ is the depth ratio, $\widehat{T}=\frac{\kappa}{\kappa_{m}}$ is the ratio of thermal diffusivity of fluid, $M=-\frac{\partial \sigma_{t}}{\partial T} \frac{\left(T_{0}-T_{u}\right) d}{\mu \kappa}$ is the Thermal Marangoni number, $M_{s 1}=-\frac{\partial \sigma_{t}}{\partial C} \frac{\left(C_{10}-C_{1 u}\right) d}{\mu \kappa}$ is the solute1 Marangoni number and $M_{s 2}=-\frac{\partial \sigma_{t}}{\partial C} \frac{\left(C_{20}-C_{2 u}\right) d}{\mu \kappa}$ is the solute2 Marangoni number.

\section{Method of Solution}

From Eqs. (47) and (51), we get velocity distributions for fluid and porous layer respectively

$$
\begin{array}{r}
W(z)=A_{1} \cosh \delta z+A_{2} \sinh \delta z \\
+A_{3} \cosh \zeta z+A_{4} \sinh \zeta z \\
W_{m}\left(z_{m}\right)=A_{5} \cosh c_{4} z_{m}+A_{6} \sinh c_{4} z_{m} \\
+A_{7} \cosh c_{5} z_{m}+A_{8} \sinh c_{5} z_{m}
\end{array}
$$

where

$\delta=\frac{\sqrt{Q}-\sqrt{Q+4 a^{2}}}{2}, \zeta=\frac{\sqrt{Q}+\sqrt{Q+4 a^{2}}}{2}, c_{4}=$ $\sqrt{\frac{c_{1}+c_{3}}{2}}, c_{5}=\sqrt{\frac{c_{1}-c_{3}}{2}}$ and $A_{i}^{\prime} s(i=1,2, \ldots .8)$ are arbitrary constants are obtained by using velocity boundary conditions of (55). The expressions for $W(z)$ and $W_{m}\left(z_{m}\right)$ are appropriately written as

$$
\begin{array}{r}
W(z)=A_{1}\left[\cosh \delta z+a_{1} \sinh \delta z\right. \\
+a_{2} \cosh \zeta z \\
\left.+a_{3} \sinh \zeta z\right] \\
W_{m}\left(z_{m}\right)=A_{1}\left[a_{4} \cosh c_{4} z_{m}+a_{5} \sinh c_{4} z_{m}\right. \\
\left.+a_{6} \cosh c_{5} z_{m}+a_{7} \sinh c_{5} z_{m}\right]
\end{array}
$$


Where

$$
\begin{gathered}
a_{1}=\frac{\Delta_{33}}{\Delta_{32}}, a_{2}=-\frac{\left(a_{1} \Delta_{30}+\Delta_{31}\right)}{\Delta_{29}}, \\
a_{3}=\frac{\left(a_{2} \Delta_{22}+\Delta_{23}-a_{1} \Delta_{21}\right)}{\Delta_{32}}, \\
a_{4}=\frac{\left(a_{2} \Delta_{18}+\Delta_{19}\right)}{\Delta_{17}}, a_{5}=\frac{\left(\widehat{T}\left(1+a_{2}\right)-a_{4} \Delta_{1}\right)}{\Delta_{2}}, \\
a_{6}=-a_{4}, a_{7}=-\frac{a_{5} c_{4}}{c_{5}} \\
\Delta_{1}=\cosh c_{4}-\cosh c_{5}, \Delta_{2}=\sinh c_{4}-\frac{c_{4}}{c_{5}} \sinh c_{5},
\end{gathered}
$$

$\Delta_{3}=c_{4} \sinh c_{4}-c_{5} \sinh c_{5}, \Delta_{4}=c_{4} \cosh c_{4}-c_{4} \cosh c_{5}$,

$\Delta_{5}=\widehat{\mu}\left[\left(c_{4}^{2}+a_{m}^{2}\right) \cosh c_{4}-\left(c_{5}^{2}+a_{m}^{2}\right) \cosh c_{5}\right]$,

$\Delta_{6}=\widehat{\mu}\left[\left(c_{4}^{2}+a_{m}^{2}\right) \sinh c_{4}-\left(c_{5}^{2}+a_{m}^{2}\right) \frac{c_{4}}{c_{5}} \sinh c_{5}\right]$,

$\Delta_{7}=\widehat{T} \beta^{2} \widehat{d}^{3}\left(\delta^{3}-3 a^{2} \delta\right), \Delta_{8}=\widehat{T} \beta^{2} \widehat{d^{3}}\left(\zeta^{3}-3 a^{2} \zeta\right)$,

$\Delta_{9}=\left(-1-3 a_{m}^{2} \widehat{\mu} \beta^{2}\right)\left(c_{4} \sinh c_{4}-c_{5} \sinh c_{5}\right)$,

$\Delta_{10}=\left(-1-3 a_{m}^{2} \widehat{\mu} \beta^{2}\right)\left(c_{4} \cosh c_{4}-c_{4} \cosh c_{5}\right)$,

$\Delta_{11}=\widehat{\mu} \beta^{2}\left(c_{4}^{3} \sinh c_{4}-c_{5}^{3} \sinh c_{5}\right)$,

$\Delta_{12}=\widehat{\mu} \beta^{2}\left(c_{4}^{3} \cosh c_{4}-c_{4} c_{5}^{2} \cosh c_{5}\right)$,

$\Delta_{13}=\Delta_{9}+\Delta_{11}, \Delta_{14}=\Delta_{10}+\Delta_{12}$,

$\Delta_{15}=\widehat{T} \widehat{d^{2}}\left(\delta^{2}+a^{2}\right), \Delta_{16}=\widehat{T} \widehat{d^{2}}\left(\zeta^{2}+a^{2}\right)$,

$\Delta_{17}=\Delta_{5}-\frac{\Delta_{1} \Delta_{6}}{\Delta_{2}}, \Delta_{18}=\Delta_{16}-\widehat{T} \frac{\Delta_{6}}{\Delta_{2}}$,

$\Delta_{19}=\Delta_{15}-\widehat{T} \frac{\Delta_{6}}{\Delta_{2}}, \Delta_{20}=\widehat{T} \widehat{d} \zeta$,

$\Delta_{21}=\widehat{T} \widehat{d} \delta, \Delta_{22}=\frac{\Delta_{18} \Delta_{3}}{\Delta_{17}+\widehat{T} \frac{\Delta_{4}}{\Delta_{2}}}-\frac{\Delta_{18} \Delta_{1} \Delta_{4}}{\Delta_{2} \Delta_{17}}$,

$\Delta_{23}=\frac{\Delta_{19} \Delta_{3}}{\Delta_{17}+\widehat{T} \frac{\Delta_{4}}{\Delta_{2}}}-\frac{\Delta_{19} \Delta_{1} \Delta_{4}}{\Delta_{2} \Delta_{17}}$,

$\Delta_{24}=\frac{\Delta_{18} \Delta_{13}}{\Delta_{17}+\widehat{T} \frac{\Delta_{14}}{\Delta_{2}}}-\frac{\Delta_{18} \Delta_{1} \Delta_{14}}{\Delta_{2} \Delta_{17}}$,

$\Delta_{25}=\frac{\Delta_{19} \Delta_{13}}{\Delta_{17}+\widehat{T} \frac{\Delta_{14}}{\Delta_{2}}}-\frac{\Delta_{19} \Delta_{1} \Delta_{14}}{\Delta_{2} \Delta_{17}}$,

$\Delta_{26}=\cosh \zeta+\frac{\Delta_{22}}{\Delta_{20}} \sinh \zeta, \Delta_{27}=\sinh \delta-\frac{\Delta_{21}}{\Delta_{20}} \sinh \zeta$,

$\Delta_{28}=\cosh \delta+\frac{\Delta_{23}}{\Delta_{20}} \sinh \zeta, \Delta_{29}=\frac{\Delta_{8} \Delta_{22}}{\Delta_{20}}-\Delta_{24}$,

$\Delta_{30}=-\frac{\Delta_{8} \Delta_{22}}{\Delta_{20}}+\Delta_{7}$,

$\Delta_{31}=\frac{\Delta_{8} \Delta_{23}}{\Delta_{20}}-\Delta_{25}, \Delta_{32}=-\frac{\Delta_{30} \Delta_{26}}{\Delta_{29}}+\Delta_{29}$,

$\Delta_{33}=\frac{\Delta_{26} \Delta_{31}}{\Delta_{29}}-\Delta_{28}$.
We get the species concentration for fluid layer $S_{1}, S_{2}$ from (49) and (50) also from (53) and (54) we get the species concentration for porous layer $S_{m 1}, S_{m 2}$ using the species concentration boundary conditions of (55) as

$$
\begin{array}{r}
S_{1}(z)=A_{1}\left[a_{16} \cosh a z+a_{17} \sinh a z+\frac{f_{2}(z)}{\tau_{1}}\right] \\
S_{m 1}\left(z_{m}\right)=A_{1}\left[a_{18} \cosh a_{m} z_{m}+a_{19} \sinh a_{m} z_{m}\right. \\
\left.+\frac{f_{m 2}\left(z_{m}\right)}{\tau_{m 1}}\right] \\
S_{2}(z)=A_{1}\left[a_{24} \cosh a z+a_{25} \sinh a z+\frac{f_{2}(z)}{\tau_{2}}\right] \\
S_{m 2}\left(z_{m}\right)=A_{1}\left[a_{26} \cosh a_{m} z_{m}+a_{27} \sinh a_{m} z_{m}\right. \\
\left.+\frac{f_{m 2}\left(z_{m}\right)}{\tau_{m 2}}\right]
\end{array}
$$

where

$$
\begin{aligned}
& f_{2}(z)=-\frac{\left(a_{1} \sinh \delta z+\cosh \delta z\right)}{\left(\delta^{2}-a^{2}\right)} \\
& -\frac{\left(a_{3} \sinh \zeta z+a_{2} \cosh \zeta z\right)}{\left(\zeta^{2}-a^{2}\right)} \\
& f_{m 2}\left(z_{m}\right)=-\frac{\left(a_{5} \sinh c_{4} z_{m}+a_{4} \cosh c_{4} z_{m}\right)}{\left(c_{4}^{2}-a_{m}^{2}\right)} \\
& -\frac{\left(a_{6} \cosh c_{5} z_{m}+a_{7} \sinh c_{5} z_{m}\right)}{\left(c_{5}^{2}-a_{m}^{2}\right)} \\
& a_{16}=\widehat{S_{1}}\left(a_{18} \cosh a_{m}+a_{19} \sinh a_{m}\right)-\Delta_{47}, \\
& a_{17}=\frac{1}{a}\left(a_{18} a_{m} \sinh a_{m}+a_{19} a_{m} \cosh a_{m}-\Delta_{48}\right), \\
& a_{18}=-\frac{\Delta_{51}}{\Delta_{50}}, a_{19}=\frac{\Delta_{49}}{a_{m}}, \\
& a_{24}=\widehat{S_{2}}\left(a_{26} \cosh a_{m}+a_{27} \sinh a_{m}\right)-\Delta_{59}, \\
& a_{25}=\frac{1}{a}\left(a_{26} a_{m} \sinh a_{m}+a_{27} a_{m} \cosh a_{m}-\Delta_{60}\right), \\
& a_{26}=\frac{\Delta_{63}}{\Delta_{62}}, a_{27}=\frac{\Delta_{61}}{a_{m}}, \\
& \Delta_{46}=\frac{1}{\tau_{1}}\left[\frac{\delta\left(\sinh \delta+a_{1} \cosh \delta\right)}{\left(\delta^{2}-a^{2}\right)}+\frac{\zeta\left(a_{2} \sinh \zeta+a_{3} \cosh \zeta\right)}{\left(\zeta^{2}-a^{2}\right)}\right], \\
& \Delta_{47}=\frac{\widehat{S_{1}}}{\tau_{m 1}}\left[\Delta_{470}\right]-\frac{1}{\tau_{1}}\left[\Delta_{471}\right], \\
& \Delta_{470}=\frac{\left(a_{5} \sinh c_{4}+a_{4} \cosh c_{4}\right)}{\left(c_{4}^{2}-a_{m}^{2}\right)} \\
& +\frac{\left(a_{7} \sinh c_{5}+a_{6} \cosh c_{5}\right)}{\left(c_{5}^{2}-a_{m}^{2}\right)}, \\
& \Delta_{471}=\frac{1}{\left(\delta^{2}-a^{2}\right)}+\frac{a_{2}}{\left(\zeta^{2}-a^{2}\right)}, \\
& \Delta_{48}=\frac{1}{\tau_{m 1}}\left[\Delta_{480}\right]-\frac{1}{\tau_{1}}\left[\Delta_{481}\right], \\
& \Delta_{480}=\frac{c_{4}\left(a_{4} \sinh c_{4}+a_{5} \cosh c_{4}\right)}{\left(c_{4}^{2}-a_{m}^{2}\right)} \\
& +\frac{c_{5}\left(a_{6} \sinh c_{5}+a_{7} \cosh c_{5}\right)}{\left(c_{5}^{2}-a_{m}^{2}\right)}, \\
& \Delta_{481}=\frac{a_{1} \delta}{\left(\delta^{2}-a^{2}\right)}+\frac{a_{3} \zeta}{\left(\zeta^{2}-a^{2}\right)}, \\
& \Delta_{49}=\frac{1}{\tau_{m 1}}\left[\frac{a_{5} c_{4}}{\left(c_{4}^{2}-a_{m}^{2}\right)}+\frac{c_{5} a_{7}}{\left(c_{5}^{2}-a_{m}^{2}\right)}\right],
\end{aligned}
$$


$\Delta_{50}=a \widehat{S_{1}} \sinh a \cosh a_{m}+a_{m} \cosh a \sinh a_{m}$,

$\Delta_{51}=\frac{\Delta_{49}}{a_{m}}\left(a \widehat{S_{1}} \sinh a \sinh a_{m}+a_{m} \cosh a \cosh a_{m}\right)-$ $\Delta_{510}$

$\Delta_{510}=\left(a \sinh a \Delta_{47}+\Delta_{48} \cosh a+\Delta_{46}\right), \Delta_{58}=\frac{\tau_{1} \Delta_{46}}{\tau_{2}}$,

$\Delta_{59}=\frac{\widehat{S_{2}}}{\tau_{m 2}}\left[\Delta_{470}\right]-\frac{1}{\tau_{2}}\left[\Delta_{471}\right]$,

$\Delta_{60}=\frac{1}{\tau_{m 2}}\left[\Delta_{480}\right]-\frac{1}{\tau_{2}}\left[\Delta_{481}\right]$,

$\Delta_{61}=\frac{1}{\tau_{m 2}}\left[\frac{a_{5} c_{4}}{\left(c_{4}^{2}-a_{m}^{2}\right)}+\frac{c_{5} a_{7}}{\left(c_{5}^{2}-a_{m}^{2}\right)}\right]$,

$\Delta_{62}=a \widehat{S_{2}} \sinh a \cosh a_{m}+a_{m} \cosh a \sinh a_{m}$,

$\Delta_{63}=-\frac{\Delta_{61}}{a_{m}}\left(a \widehat{S_{2}} \sinh a \sinh a_{m}+a_{m} \cosh a \cosh a_{m}\right)+$ $\Delta_{630}$

$\Delta_{630}=a \sinh a \Delta_{59}+\Delta_{60} \cosh a+\Delta_{58}$.

\subsection{Linear temperature profile}

Here taking

$$
h(z)=h_{m}\left(z_{m}\right)=1
$$

Substituting equation (64) into (48) and (52), we get temperature distributions for fluid and porous layers using temperature boundary conditions of (55) and they are

$$
\begin{array}{r}
\theta(z)=A_{1}\left[a_{20} \cosh a z+a_{21} \sinh a z+g_{1}(z)\right] \\
\theta_{m}\left(z_{m}\right)=A_{1}\left[a_{22} \cosh a_{m} z_{m}+a_{23} \sinh a_{m} z_{m}\right. \\
\left.+g_{m 1}\left(z_{m}\right)\right]
\end{array}
$$

Where

$$
\begin{aligned}
& g_{1}(z)=-\frac{\left(a_{1} \sinh \delta z+\cosh \delta z\right)}{\left(\delta^{2}-a^{2}\right)} \\
& -\frac{\left(a_{3} \sinh \zeta z+a_{2} \cosh \zeta z\right)}{\zeta^{2}-a^{2}} \\
& g_{m 1}\left(z_{m}\right)=-\frac{\left(a_{5} \sinh c_{4} z_{m}+a_{4} \cosh c_{4} z_{m}\right)}{\left(c_{4}^{2}-a_{m}^{2}\right)} \\
& -\frac{\left(a_{7} \sinh c_{5} z_{m}+a_{6} \cosh c_{5} z_{m}\right)}{\left(c_{5}^{2}-a_{m}^{2}\right)} \\
& a_{20}=\widehat{T}\left(a_{22} \cosh a_{m}+a_{23} \sinh a_{m}\right)-\Delta_{52}, \\
& a_{21}=\frac{1}{a}\left(a_{22} a_{m} \sinh a_{m}+a_{23} a_{m} \cosh a_{m}-\Delta_{53}\right) \\
& a_{22}=-\frac{\Delta_{57}}{\Delta_{56}}, a_{23}=\frac{\Delta_{55}}{a_{m}} . \\
& \Delta_{52}=\widehat{T} \Delta_{480}-\Delta_{470}, \Delta_{53}=\Delta_{480}-\Delta_{481}, \\
& \Delta_{54}=\frac{\delta\left(\sinh \delta+a_{1} \cosh \delta\right)}{\left(\delta^{2}-a^{2}\right)}+\frac{\zeta\left(a_{2} \sinh \zeta+a_{3} \cosh \zeta\right)}{\left(\zeta^{2}-a^{2}\right)}
\end{aligned}
$$

$\Delta_{55}=\frac{a_{5} c_{4}}{\left(c_{4}^{2}-a_{m}^{2}\right)^{2}}+\frac{c_{5} a_{7}}{\left(c_{5}^{2}-a_{m}^{2}\right)^{2}}, \Delta_{56}=\Delta_{38}$,

$\Delta_{57}=\frac{\Delta_{55}}{a_{m}}\left(a \widehat{T} \sinh a \sinh a_{m}+a_{m} \cosh a \cosh a_{m}\right)-\Delta_{570}$,

$\Delta_{570}=a \sinh a \Delta_{52}+\Delta_{53} \cosh a+\Delta_{54}$.

The thermal Marangoni number obtained from (55) and is found to be

$$
M=\frac{-\left[D^{2} W(1)+M_{s 1} a^{2} S_{1}(1)+M_{s 2} a^{2} S_{2}(1)\right]}{a^{2} \theta(1)}
$$

For this model

$$
M_{1}=-\frac{\left(\Lambda_{1}+\Lambda_{2}+\Lambda_{3}\right)}{\Lambda_{4}}
$$

Where

$$
\Lambda_{1}=\delta^{2} \cosh \delta+a_{1} \delta^{2} \sinh \delta+a_{2} \zeta^{2} \cosh \zeta+a_{3} \zeta^{2} \sinh \zeta
$$

$\Lambda_{2}=M_{s 1} a^{2}\left[a_{16} \cosh a+a_{17} \sinh a-\frac{1}{\tau_{1}} R_{1}\right]$,

$\Lambda_{3}=M_{s 2} a^{2}\left[a_{24} \cosh a+a_{25} \sinh a-\frac{1}{\tau_{2}} R_{1}\right]$,

$\Lambda_{4}=a^{2}\left[a_{20} \cosh a+a_{21} \sinh a-R_{1}\right]$,

$R_{1}=\frac{\left(a_{1} \sinh \delta+\cosh \delta\right)}{\left(\delta^{2}-a^{2}\right)}+\frac{\left(a_{3} \sinh \zeta+a_{2} \cosh \zeta\right)}{\left(\zeta^{2}-a^{2}\right)}$.

\subsection{Parabolic temperature profile}

We consider the profile as following Sparrow et al. [20],

$$
h(z)=2 z \quad \text { and } \quad h_{m}\left(z_{m}\right)=2 z_{m}
$$

Substituting equation (68) into (48) and (52), we get temperature distributions for fluid and porous layers using temperature boundary conditions of (55) and they are

$$
\begin{array}{r}
\theta(z)=A_{1}\left[a_{8} \cosh a z+a_{9} \sinh a z+f_{1}(z)\right] \\
\theta_{m}\left(z_{m}\right)=A_{1}\left[a_{10} \cosh a_{m} z_{m}+a_{11} \sinh a_{m} z_{m}\right. \\
\left.+f_{m 1}\left(z_{m}\right)\right]
\end{array}
$$

where

$$
\begin{aligned}
& f_{1}(z)=-\left(R_{2}+R_{3}\right), f_{m 1}\left(z_{m}\right)=-\left(R_{4}+R_{5}\right) \\
& R_{2}=\frac{2 z\left(a_{1} \sinh \delta z+\cosh \delta z\right)}{\left(\delta^{2}-a^{2}\right)}-\frac{4 \delta\left(\sinh \delta z+a_{1} \cosh \delta z\right)}{\left(\delta^{2}-a^{2}\right)^{2}} \\
& R_{3}=\frac{2 z\left(a_{3} \sinh \zeta z+a_{2} \cosh \zeta z\right)}{\left(\zeta^{2}-a^{2}\right)} \\
& -\frac{4 \zeta\left(a_{2} \sinh \zeta z+a_{3} \cosh \zeta z\right)}{\left(\zeta^{2}-a^{2}\right)^{2}} \\
& R_{4}=\frac{2 z_{m}\left(a_{5} \sinh c_{4} z_{m}+a_{4} \cosh c_{4} z_{m}\right)}{\left(c_{4}^{2}-a_{m}^{2}\right)} \\
& -\frac{4 c_{4}\left(a_{4} \sinh c_{4} z_{m}+a_{5} \cosh c_{4} z_{m}\right)}{\left(c_{4}^{2}-a_{m}^{2}\right)^{2}}
\end{aligned}
$$




$$
\begin{aligned}
& R_{5}=\frac{2 z_{m}\left(a_{7} \sinh c_{5} z_{m}+a_{6} \cosh c_{5} z_{m}\right)}{\left(c_{5}^{2}-a_{m}^{2}\right)} \\
& -\frac{4 c_{5}\left(a_{6} \sinh c_{5} z_{m}+a_{7} \cosh c_{5} z_{m}\right)}{\left(c_{5}^{2}-a_{m}^{2}\right)^{2}} \\
& a_{8}=\widehat{T}\left(a_{10} \cosh a_{m}+a_{11} \sinh a_{m}\right)+\Delta_{35}, \\
& a_{9}=\frac{1}{a}\left(a_{10} a_{m} \sinh a_{m}+a_{11} a_{m} \cosh a_{m}+\Delta_{36}\right), \\
& a_{10}=\frac{\Delta_{39}}{\Delta_{38}}, a_{11}=-\frac{\Delta_{37}}{a_{m}}, \\
& \Delta_{34}=\frac{2\left(\delta^{2}+a^{2}\right)}{\left(\delta^{2}-a^{2}\right)^{2}}\left(a_{1} \sinh \delta+\cosh \delta\right)+\Delta_{340}+\Delta_{341}, \\
& \Delta_{340}=-\frac{2}{\left(\zeta^{2}-a^{2}\right)}\left[\left(a_{3}+a_{2} \zeta\right) \sinh \zeta+\left(a_{2}+a_{3} \zeta\right) \cosh \zeta\right], \\
& \Delta_{341}=-\frac{2 \delta}{\left(\delta^{2}-a^{2}\right)}\left(\sinh \delta+a_{1} \cosh \delta\right)+\Delta_{342}, \\
& \Delta_{342}=\frac{4 \zeta^{2}}{\left(\zeta^{2}-a^{2}\right)^{2}}\left(a_{3} \sinh \zeta+a_{2} \cosh \zeta\right), \\
& \Delta_{35}=4 \widehat{T} \Delta_{480}-4 \Delta_{481}-\Delta_{351}, \\
& \Delta_{351}=2 \widehat{T}\left[\frac{\left(a_{5} \sinh c_{4}+a_{4} \cosh c_{4}\right)}{\left(c_{4}^{2}-a_{m}^{2}\right)}\right. \\
& \left.+\frac{\left(a_{7} \sinh c_{5}+a_{6} \cosh c_{5}\right)}{\left(c_{5}^{2}-a_{m}^{2}\right)}\right], \\
& \Delta_{36}=-\frac{2\left(\delta^{2}+a^{2}\right)}{\left(\delta^{2}-a^{2}\right)^{2}}-2 a_{2} \frac{\left(\zeta^{2}+a^{2}\right)}{\left(\zeta^{2}-a^{2}\right)^{2}}+\Delta_{360}+\Delta_{361}, \\
& \Delta_{360}=2 \Delta_{362}+2 \Delta_{363}, \Delta_{361}=2 \Delta_{364}+2 \Delta_{365}, \\
& \Delta_{362}=-\frac{c_{4}}{\left(c_{4}^{2}-a_{m}^{2}\right)}\left(a_{4} \sinh c_{4}+a_{5} \cosh c_{4}\right), \\
& \Delta_{363}=\frac{\left(c_{4}^{2}+a_{m}^{2}\right)}{\left(c_{4}^{2}-a_{m}^{2}\right)^{2}}\left(a_{5} \sinh c_{4}+a_{4} \cosh c_{4}\right), \\
& \Delta_{364}=-\frac{c_{5}}{\left(c_{5}^{2}-a_{m}^{2}\right)}\left(a_{6} \sinh c_{5}+a_{7} \cosh c_{5}\right), \\
& \Delta_{365}=\frac{\left(c_{5}^{2}+a_{m}^{2}\right)}{\left(c_{5}^{2}-a_{m}^{2}\right)^{2}}\left(a_{7} \sinh c_{5}+a_{6} \cosh c_{5}\right), \\
& \Delta_{37}=2 a_{6} \frac{\left(c_{5}^{2}+a_{m}^{2}\right)}{\left(c_{5}^{2}-a_{m}^{2}\right)^{2}}+2 a_{4} \frac{\left(c_{4}^{2}+a_{m}^{2}\right)}{\left(c_{4}^{2}-a_{m}^{2}\right)^{2}}, \\
& \Delta_{38}=a \widehat{T} \sinh a \cosh a_{m}+a_{m} \cosh a \sinh a_{m}, \\
& \Delta_{39}=\frac{\Delta_{37}\left(a \widehat{T} \sinh a \sinh a_{m}\right)}{a_{m}}+\Delta_{390}-\Delta_{391}, \\
& \Delta_{390}=\Delta_{37} \cosh a \cosh a_{m}, \\
& \Delta_{391}=\left(a \sinh a \Delta_{35}+\Delta_{36} \cosh a+\Delta_{34}\right)
\end{aligned}
$$

The thermal Marangoni number for this model obtained from (55) and is found to be

$$
M_{2}=-\frac{\left(\Lambda_{1}+\Lambda_{2}+\Lambda_{3}\right)}{\Lambda_{5}}
$$

where

$$
\begin{aligned}
\Lambda_{5} & =\left[a_{8} \cosh a+a_{9} \sinh a+R_{6}+R_{7}\right] \\
R_{6} & =-\frac{2\left(a_{1} \sinh \delta+\cosh \delta\right)}{\left(\delta^{2}-a^{2}\right)}+\frac{4 \delta\left(\sinh \delta+a_{1} \cosh \delta\right)}{\left(\delta^{2}-a^{2}\right)^{2}},
\end{aligned}
$$

$$
\begin{aligned}
& R_{7}=-\frac{2\left(a_{3} \sinh \zeta+a_{2} \cosh \zeta\right)}{\left(\zeta^{2}-a^{2}\right)} \\
& +\frac{4 \zeta\left(a_{2} \sinh \zeta+a_{3} \cosh \zeta\right)}{\left(\zeta^{2}-a^{2}\right)^{2}} .
\end{aligned}
$$

\subsection{Inverted parabolic temperature profile}

We have

$$
h(z)=2(1-z) \quad \text { and } \quad h_{m}\left(z_{m}\right)=2\left(1-z_{m}\right)
$$

Substituting equation (72) into (48) and (52), we get temperature distributions for fluid and porous layers using temperature boundary conditions of (55) and they are

$$
\begin{array}{r}
\theta(z)=A_{1}\left[a_{12} \cosh a z+a_{13} \sinh a z+f_{3}(z)\right] \\
\theta_{m}\left(z_{m}\right)=A_{1}\left[a_{14} \cosh a_{m} z_{m}+a_{15} \sinh a_{m} z_{m}\right. \\
\left.+f_{m 3}\left(z_{m}\right)\right]
\end{array}
$$

Where

$$
\begin{aligned}
& f_{3}(z)=R_{8}+R_{9}, f_{m 3}\left(z_{m}\right)=-2\left(1-z_{m}\right) R_{10}-R_{11}, \\
& R_{8}=-\frac{2(1-z)\left(a_{1} \sinh \delta z+\cosh \delta z\right)}{\left(\delta^{2}-a^{2}\right)} \\
& -\frac{4 \delta\left(\sinh \delta z+a_{1} \cosh \delta z\right)}{\left(\delta^{2}-a^{2}\right)^{2}} \\
& R_{9}=-\frac{2(1-z)\left(a_{3} \sinh \zeta z+a_{2} \cosh \zeta z\right)}{\left(\zeta^{2}-a^{2}\right)} \\
& -\frac{4 \zeta\left(a_{2} \sinh \zeta z+a_{3} \cosh \zeta z\right)}{\left(\zeta^{2}-a^{2}\right)^{2}} \\
& R_{10}=\frac{\left(a_{5} \sinh c_{4} z_{m}+a_{4} \cosh c_{4} z_{m}\right)}{\left(c_{4}^{2}-a_{m}^{2}\right)} \\
& +\frac{\left(a_{7} \sinh c_{5} z_{m}+a_{6} \cosh c_{5} z_{m}\right)}{\left(c_{5}^{2}-a_{m}^{2}\right)} \\
& R_{11}=-\frac{4 c_{4}\left(a_{4} \sinh c_{4} z_{m}+a_{5} \cosh c_{4} z_{m}\right)}{\left(c_{4}^{2}-a_{m}^{2}\right)^{2}}-R_{111}, \\
& R_{111}=\frac{4 c_{5}\left(a_{6} \sinh c_{5} z_{m}+a_{7} \cosh c_{5} z_{m}\right)}{\left(c_{5}^{2}-a_{m}^{2}\right)^{2}} \\
& a_{12}=\widehat{T}\left(a_{14} \cosh a_{m}+a_{15} \sinh a_{m}\right)+\Delta_{41}, \\
& a_{13}=\frac{1}{a}\left(a_{14} a_{m} \sinh a_{m}+a_{15} a_{m} \cosh a_{m}+\Delta_{42}\right) \text {, } \\
& a_{14}=\frac{\Delta_{45}}{\Delta_{44}}, a_{15}=-\frac{\Delta_{43}}{a_{m}}, \\
& \Delta_{40}=-\frac{2\left(\delta^{2}+a^{2}\right)}{\left(\delta^{2}-a^{2}\right)^{2}}\left(a_{1} \sinh \delta+\cosh \delta\right)-\Delta_{400}, \\
& \Delta_{400}=\frac{2\left(\zeta^{2}+a^{2}\right)}{\left(\zeta^{2}-a^{2}\right)^{2}}\left(a_{3} \sinh \zeta+a_{2} \cosh \zeta\right), \\
& \Delta_{41}=\frac{2\left(\delta^{2}-a^{2}\right)+4 a_{1} \delta}{\left(\delta^{2}-a^{2}\right)^{2}}+\frac{2 a_{2}}{\left(\zeta^{2}-a^{2}\right)}+\Delta_{410}-\Delta_{411}, \\
& \Delta_{410}=\frac{4 a_{3} \zeta}{\left(\zeta^{2}-a^{2}\right)^{2}}-\frac{4 c_{4} \widehat{T}}{\left(c_{4}^{2}-a_{m}^{2}\right)^{2}}\left(a_{4} \sinh c_{4}+a_{5} \cosh c_{4}\right), \\
& \Delta_{411}=\frac{4 c_{5} \widehat{T}}{\left(c_{5}^{2}-a_{m}^{2}\right)^{2}}\left(a_{6} \sinh c_{5}+a_{7} \cosh c_{5}\right),
\end{aligned}
$$


$\Delta_{42}=\frac{2 a_{1} \delta\left(\delta^{2}-a^{2}\right)+2\left(\delta^{2}+a^{2}\right)}{\left(\delta^{2}-a^{2}\right)^{2}}+\Delta_{420}$,

$\Delta_{420}=\frac{2 a_{3} \zeta}{\left(\zeta^{2}-a^{2}\right)}+2 a_{2} \frac{\left(\zeta^{2}+a^{2}\right)}{\left(\zeta^{2}-a^{2}\right)^{2}}-2 R_{12}$,

$R_{12}=\Delta_{363}+\Delta_{365}$,

$\Delta_{43}=-\frac{2 c_{4} a_{5}}{\left(c_{4}^{2}-a_{m}^{2}\right)}-\frac{2 c_{5} a_{7}}{\left(c_{5}^{2}-a_{m}^{2}\right)}-\frac{2 a_{4}\left(c_{4}^{2}+a_{m}^{2}\right)}{\left(c_{4}^{2}-a_{m}^{2}\right)^{2}}-$

$\frac{2 a_{6}\left(c_{5}^{2}+a_{m}^{2}\right)}{\left(c_{5}^{2}-a_{m}^{2}\right)^{2}}, \Delta_{44}=\Delta_{38}$

$\Delta_{45}=\frac{\Delta_{43}}{a_{m}}\left(a \widehat{T} \sinh a \sinh a_{m}+a_{m} \cosh a \cosh a_{m}\right)-\Delta_{450}$,

$\Delta_{450}=\left(a \sinh a \Delta_{41}+\Delta_{42} \cosh a+\Delta_{40}\right)$.

The thermal Marangoni number for this model obtained from (55) and is found to be

$$
M_{3}=-\frac{\left(\Lambda_{1}+\Lambda_{2}+\Lambda_{3}\right)}{\Lambda_{6}}
$$

Where

$$
\begin{aligned}
\Lambda_{6} & =\left[a_{12} \cosh a+a_{13} \sinh a-R_{13}\right] \\
R_{13} & =\frac{4 \delta\left(\sinh \delta+a_{1} \cosh \delta\right)}{\left(\delta^{2}-a^{2}\right)^{2}}+\frac{4 \zeta\left(a_{2} \sinh \zeta+a_{3} \cosh \zeta\right)}{\left(\zeta^{2}-a^{2}\right)^{2}}
\end{aligned}
$$

\section{Results and Discussion}

The thermal Marangoni numbers $M_{1}$ for linear, $M_{2}$ for parabolic and $M_{3}$ for inverted parabolic temperature profiles are obtained for diffrent physical parameters and the constraints are drawn against the depth ratio $\widehat{d}=\frac{d_{m}}{d}$. The dimensionless fixed values are $\widehat{T}=1.0, a=1.2, \beta=0.3, \widehat{\mu}=2.0, \epsilon=1.0, Q=50, M_{s 1}=$ $M_{s 2}=10, \tau_{1}=\tau_{2}=\tau_{m 1}=\tau_{m 2}=\widehat{S_{1}}=\widehat{S_{2}}=0.75$.

The effects of the parameters $a, \beta, \epsilon, \widehat{\mu}, Q, M_{s 1}, M_{s 2}$ and $\tau_{1}$ on all the three thermal Marangoni numbers are depicted in Figs. 2 to 9. The main observation that out of three profiles the parabolic profile is the most stable one and the linear profile is the most unstable one as the thermal Marangoni numbers are highest and lowest respectively, for a given set of fixed values of parameters, specially for porous layer dominant systems. For fluid layer dominant system, there is no much change in the thermal Marangoni numbers for all the profiles.

The variations of $a$, horizontal wave number on the thermal Marangoni numbers $M_{1}, M_{2}$ and $M_{3}$ are respectively shown in Figs. 2(a)-(c) for $a=1.3,1.4$ and 1.5. We observed that the thermal Marangoni number for the parabolic profile is larger than those for the linear and inverted parabolic profiles. For all the profiles, it is evident from the graph that an increase in the value of $a$, the thermal Marangoni number decreases and its effect is to destabilize the system.

The variations of the porous parameter $\beta$ on the three thermal
Marangoni numbers are depicted Figs. 3(a)-(c). The curves for $\beta=0.2,0.3,0.4$. Increase in the value of $\beta$, that is, increasing the permeability, the thermal Marangoni numbers increase for all the three profiles. Hence the surface tension driven triple diffusive magneto convection occurs faster on increasing the porous parameter, which is physically reasonable, as there is no more way for the fluid to move. So, the system is stabilized. Figs. 4 (a)-(c) show the effects of porosity $\epsilon$ for the values $\epsilon=0.8,0.9,1.0$. There is no effect of porosity up to the value $\widehat{d}=0.1$. At $\widehat{d} \geq 0.2$ the curves are diverging and also whereas $\epsilon$ increases, the thermal Marangoni number increases i.e.to stabilize the system.

Figs. 5(a)-(c) show the variations of viscosity ratio $\widehat{\mu}$ for the values $\widehat{\mu}=2.5,3.0,3.5$. Increase in the value of $\widehat{\mu}$, the values of the thermal Marangoni numbers $M_{1}, M_{2}$ and $M_{3}$ increases for $\widehat{d} \leq 0.4$. Also, $\widehat{d} \geq 0.4$ the increase in the values of viscosity ratio decreases the thermal Marangoni numbers for all the profiles. By increasing the viscosity ratio the system can stabilized or destabilize the system and hence the surface tension driven triple diffusive magneto convection is delayed or faster.

Figs. 6(a)-(c) illustrates the variations of the Chandrasekhar number $Q$, for the values $Q=50,60,70$. We observed that the thermal Marangoni number for the parabolic profile is more stable than the other profiles. For all the profiles, when the value of the $Q$ is increased, the thermal Marangoni numbers increases and hence stabilize the system, as expected. Figs. 7(a)-(c) show the effects of the $M_{s 1}$ is the solute1 Marangoni number for $M_{s 1}=10,20,30$. By increasing the values of solute1 Marangoni numbers, the thermal Marangoni numbers increase for all the three temperature profiles. Hence its effect is to stabilize the system. Linear profile is suitable for fluid layer dominant composite layer. Whereas parabolic and inverted parabolic profiles are suitable for porous layer dominant composite layer.

Figs. 8 (a)-(c) illustrates the effects of the $M_{s 2}$ is the solute2 Marangoni number for $M_{s 2}=100,300,500$. By increasing the values of $M_{s 2}$, the thermal Marangoni numbers decrease for all the three temperature profiles. So, the surface tension driven triple diffusive magneto convection can be preponed by increasing solute2 Marangoni number, hence the system can be destabilized.

Figs. 9 (a)-(c) display the effects of $\tau_{1}$ is the ratio of solute 1 diffusivity to thermal diffusivity fluid in fluid layer for $M_{1}, M_{2}$ and $M_{3}$ respectively for the values $\tau_{1}=0.50,0.75,1.0$. For all the three profiles, there is a decrease in the values of the thermal Marangoni numbers. Increasing the value of $\tau_{1}$ the surface tension driven triple diffusive magneto convection becomes faster i.e., system can be destabilized.

\section{Conclusions}

(i) The parabolic temperature profile is the most suitable for the situations demanding the control of triple diffusive surface tension driven convection, where as the linear and inverted parabolic profile is suitable for the situations demanding convection. 
(ii) By increasing the values of $\beta, \widehat{\mu}, Q, M_{s 1}$ and by decreasing the values of $a, \epsilon, M_{s 2}$ and $\tau_{1}$ the surface tension driven triple diffusive magneto convection in a composite layer under micro gravity condition can be delayed and hence the system can be stabilized.

\section{Acknowledgements}

We are greatful to Anthony Robinson, Editorial Assistant for their appropriate and constructive suggestions to improve this work. The author Manjunatha. N, express his sincere thanks to the management of REVA University, Bengaluru for their encouragement and support.

\section{REFERENCES}

[1] Melviana Johnson Fu, Norihan Md Arifin, Roslinda Nazar and Mohd Noor Saad., Effect of non-uniform temperature gradient and magnetic field on Marangoni convection in a micropolar fluid, International review of chemical engineering, Vol.1, No. 4, pp.369-373, (2009).

[2] Isa S. P. M., Norihan Md. Arifin, Mohd Noor Saad and Roslinda Mohd Nazar., Effects of non-uniform temperature gradient on Marangoni convection with free slip condition, American Journal of Scientific Research, Vol. 1, pp.37-44, (2009).

[3] Isa S. P. M. , Arifin N. M., Nazar R. and Saad M. N., Effect of non-uniform temperature gradient and magnetic field on onset of Marangoni convection heated from below by a constant heat flux, Appl. Math. Mech.-Engl. Ed., Vol.31, pp.797-804, (2010a).

[4] Isa S. P. M. , Arifin N. M., Nazar R. and Saad M. N. Combined effect of non-uniform temperature gradient and magnetic field on Benard-Marangoni convection with a constant heat flux, J. Open Aerospace Eng., Vol. 3, pp.59-64. (2010b).

[5] Norihan Md Arifin, Siti Suzilliana Putri Mohamed Isa, and Roslinda Nazar, Effect of non-uniform temperature and magnetic field on convection driven by surface tension and buoyancy, Proceedings of the international multi conference of engineers and scientist 2011, Vol II, IMECS, Hong kong, March 16-18, (2011).

[6] Mahmud M. N., Mustafa Z. and Hashim I., Effects of control on the onset of Benard-Marangoni convection in a micropolar fluid, International Communications in Heat and Mass Transfer, Vol.37, No. 9, pp.1335-1339, (2010).

[7] Pranesh S. and Riya Baby., Effect of non-uniform temperature gradient on the onset of Rayleigh-Benard electro convection in a micropolar fluid, Applied Mathematics, Vol.3, pp.442-450, (2012).

[8] Pranesh S. and Kiran R.V., Effect of Non-Uniform Temperature Gradient on the Onset of Rayleigh-Benard-Magneto convection in Micropolar Fluid with Maxwell-Cattaneo Law, Mapana Journal of sciences, Vol.11, No. 3, pp.193-214, (2012).
[9] Thadathil Varghese Joseph, Sree Ramaiah Manjunath and Subbarama Pranesh, Effect of non-uniform basic temperature gradient on the onset of Rayleigh-Benard-Marangoni electroconvection in a micropolar fluid, Applied Mathematics, Vol. 4, No. 8, pp. 1180-1188, (2013).

[10] Azmi H. M. and Idris R., Effects of controller and nonuniform temperature profile on the onset of Rayleigh-Benard-Marangoni electroconvection in a micropolar fluid, Journal of Applied Mathematics, Vol. 2014 , pp.1-8, (2014).

[11] Sandhya S. and Sangeetha George K. , Effect of rotation and non-uniform temperature gradients on the onset of Marangoni convection in a fluid with suspended particles under microgravity condition, International Journal of Statistics and Applied Mathematics, Vol.3, No.2, Part G, pp.499-506, (2018).

[12] Shivakumara I.S., Onset of convection in a couple-stress fluidsaturated porous medium: Effects of non-uniform temperature gradients, Archive of Applied Mechanics, Vol.80, No.8, pp.949-957, (2010).

[13] Shivakumara I.S., JinhoLee, Vajravelu K. and Mamatha A.L. Effects of thermal non-equilibrium and non-uniform temperature gradients on the onset of convection in a heterogeneous porous medium, International Communications in Heat and Mass Transfer, Vol. 38, No.7, pp.906-910, (2011).

[14] Shivakumara, I.S., Sureshkumar S. and Devaraju N., Effect of non-uniform temperature gradients on the onset of convection in a couple stress fluid saturated porous medium, Journal of Applied Fluid Mechanics, Vol.5, No.1, pp.49-55, (2012).

[15] Manjunatha N. and Sumithra R., Effects of non-uniform temperature gradients on double diffusive Marangoni convection in a two layer system, International journal of Pure and Applied Mathematics, Vol.118, No. 2, pp.203-220, (2018a).

[16] Manjunatha N. and Sumithra R., Effects of non-uniform temperature gradients on surface tension driven two component magnetoconvection in a porous-fluid system, ARPN Journal of Engineering and Applied Sciences Vol.13, No. 2, pp.429-441, (2018b).

[17] Manjunatha N. and Sumithra R., Effects of non-uniform temperature gradients on triple diffusive Marangoni convection in a composite layer, Open Journal of Applied Sciences, Vol.9, No. 8, pp.640-660, (2019).

[18] Chen F. and Chen C.F., Onset of Finger convection in a horizontal porous layer underlying a fluid layer, J. Heat transfer Vol.110, pp.403, (1988).

[19] Nield D. A., Onset of convection in a fluid layer overlying a layer of a porous medium, J. Fluid mech., Vol.81, pp.513-522, (1977).

[20] Sparrow E. W., Goldstein R. J. and Jonson V. K., Thermal instability in a horizontal fluid layer: Effect of boundary conditions and nonlinear temperature profile, J. Fluid Mech., Vol. 18 , pp.513-528, (1964). 


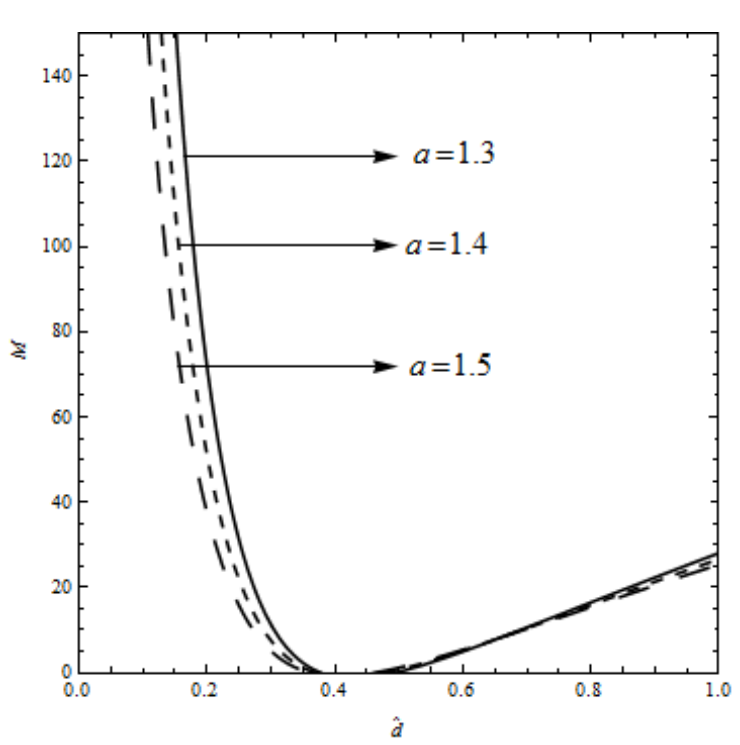

(a)

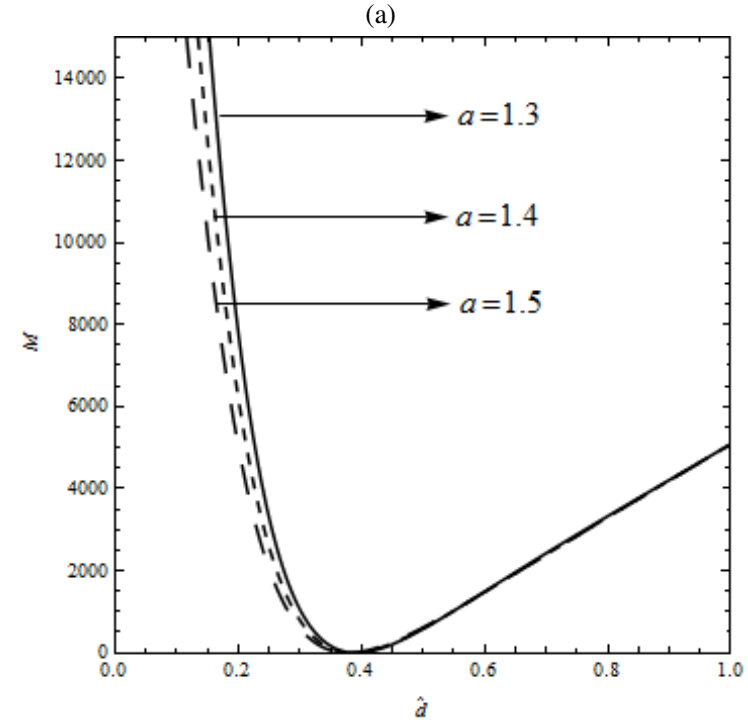

(b)

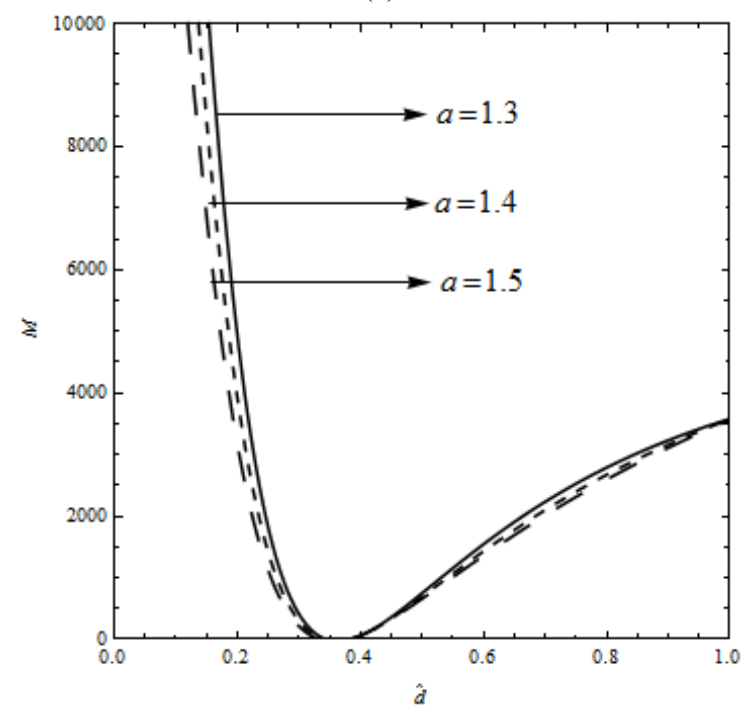

(c)

Figure 2. The effects of horizontal wave number $a$

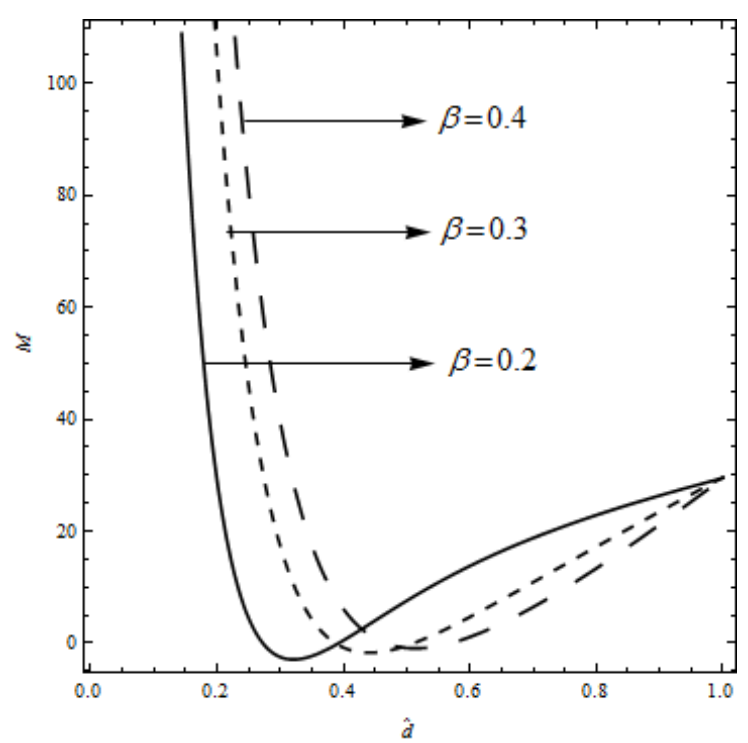

(a)

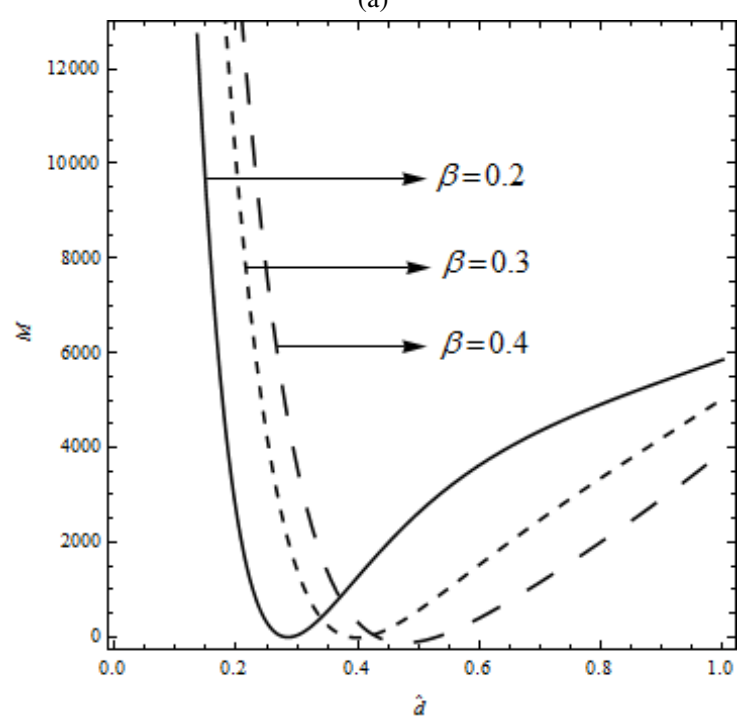

(b)

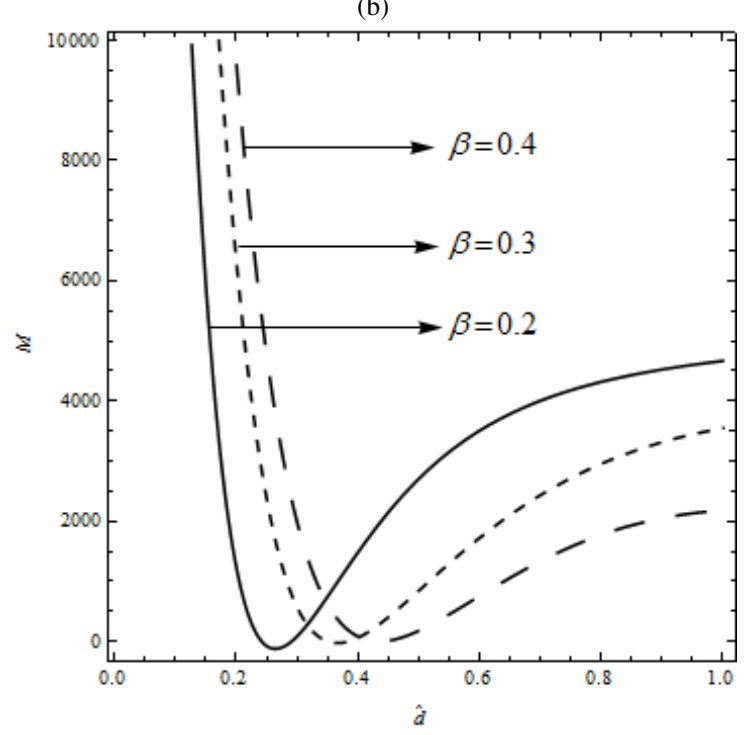

(c)

Figure 3. The effects of porous parameter $\beta$ 


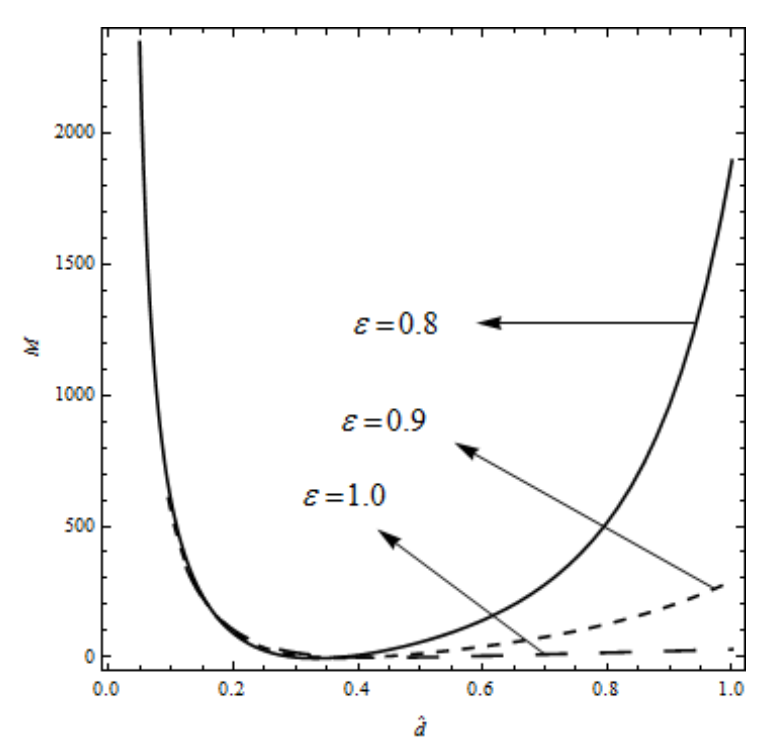

(a)

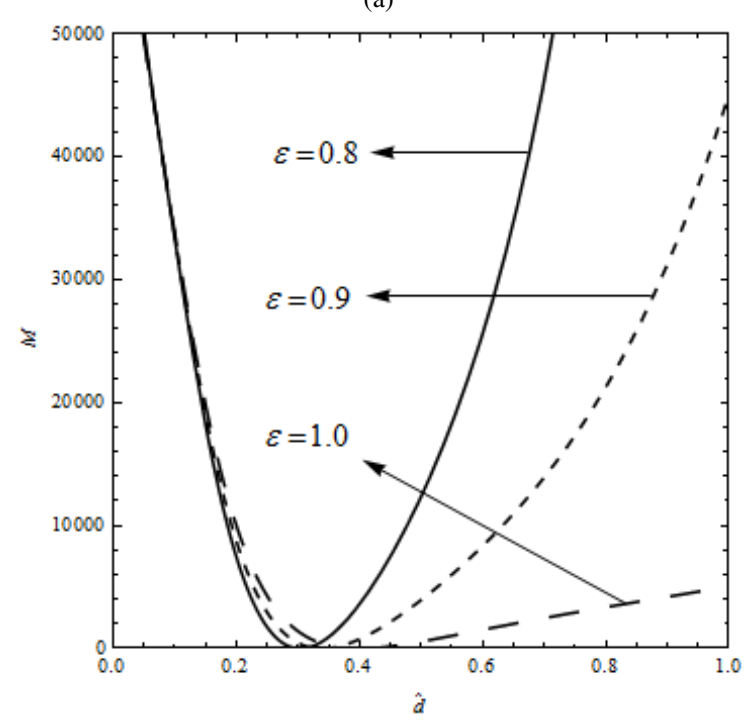

(b)

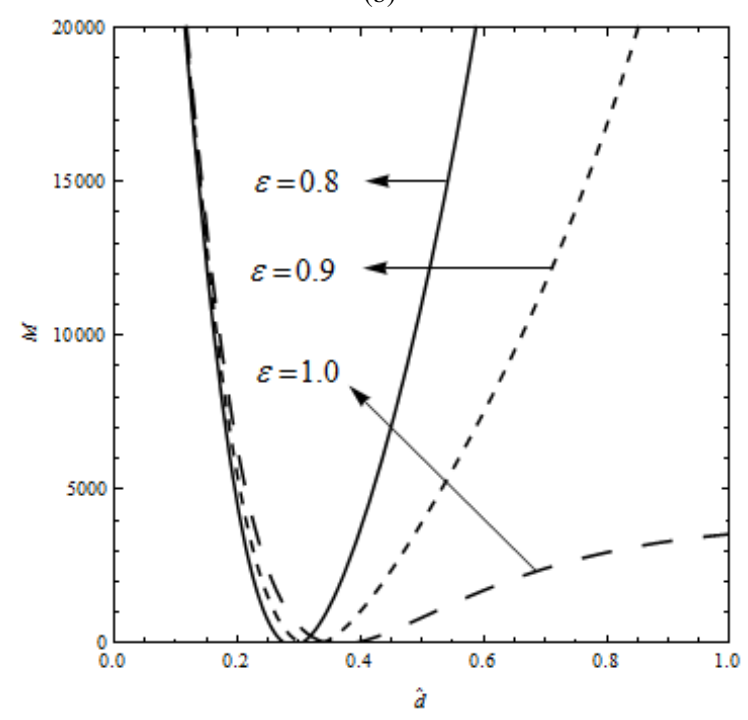

(c)

Figure 4. The effects of porosity $\epsilon$

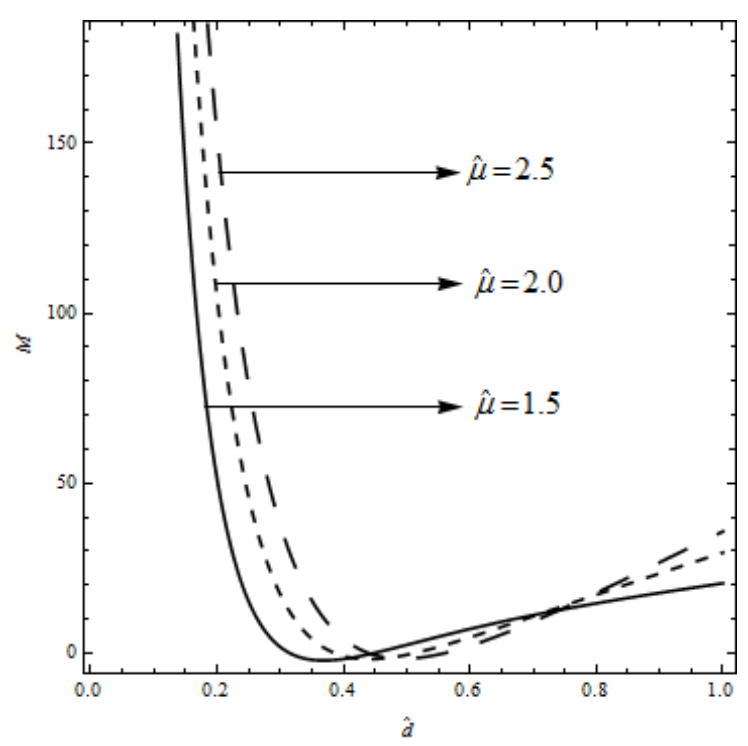

(a)

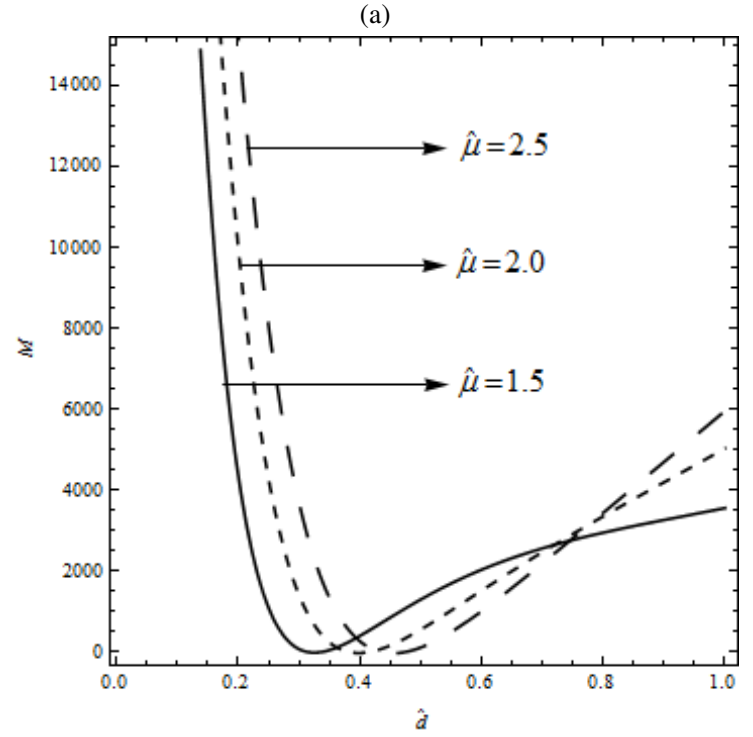

(b)

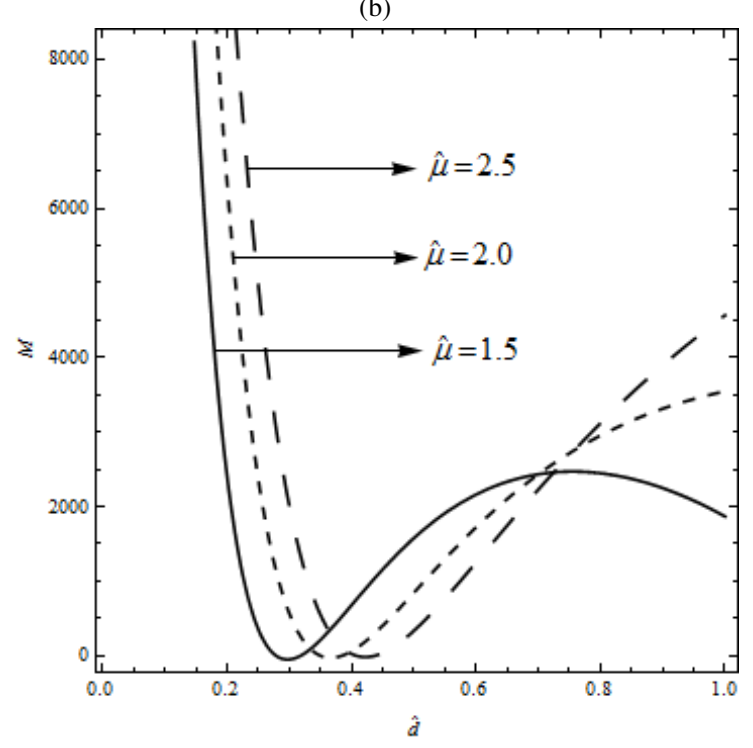

(c)

Figure 5. The effects of viscosity ratio $\widehat{\mu}$ 


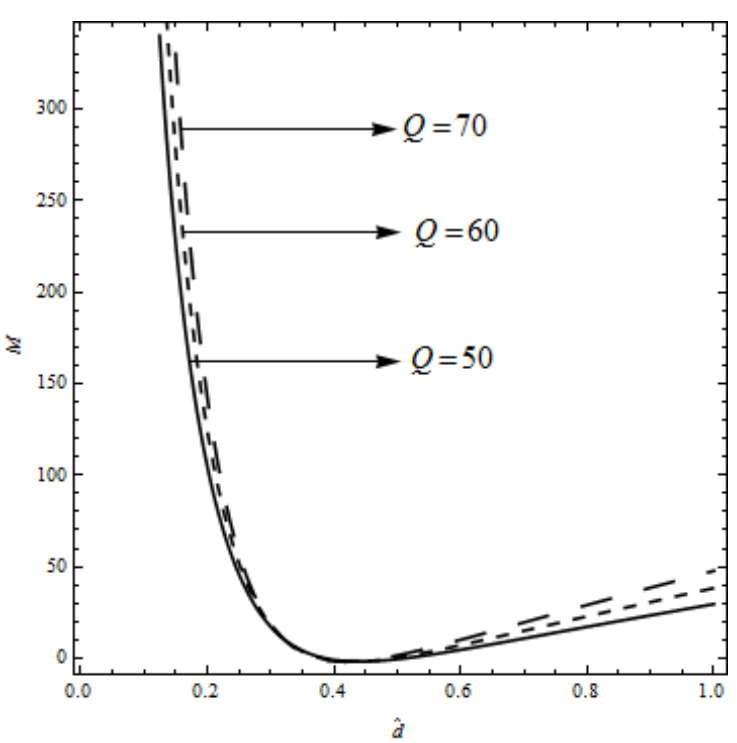

(a)

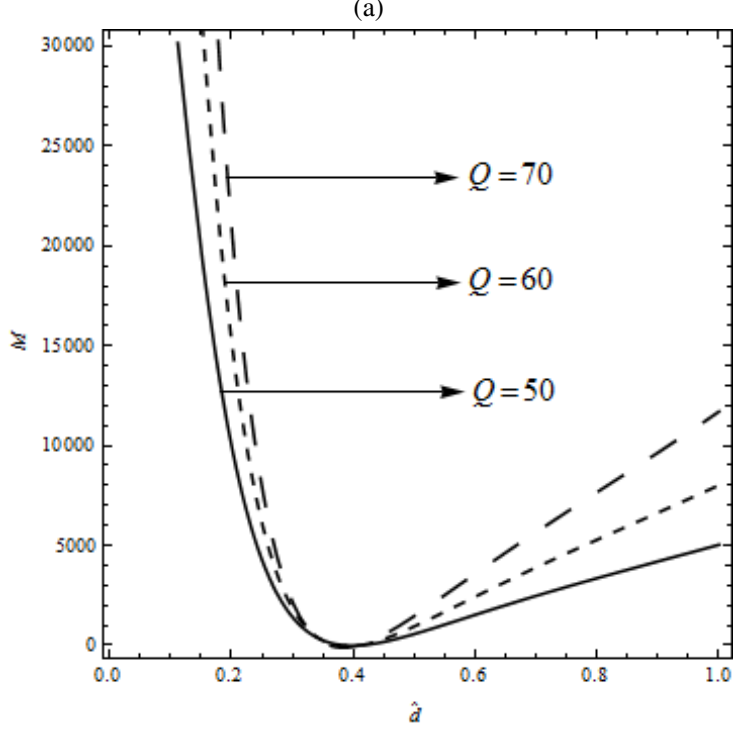

(b)

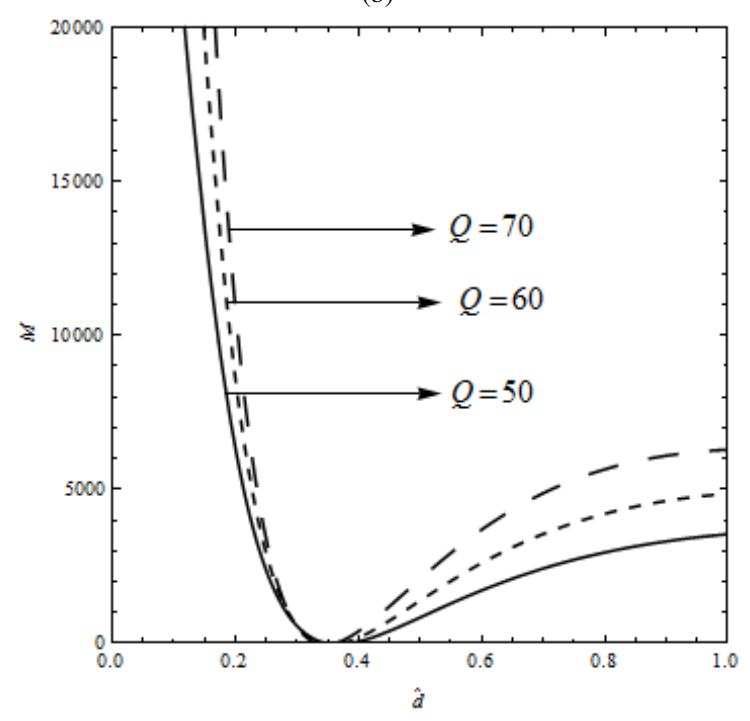

(c)

Figure 6. The effects of Chandrasekhar number $Q$

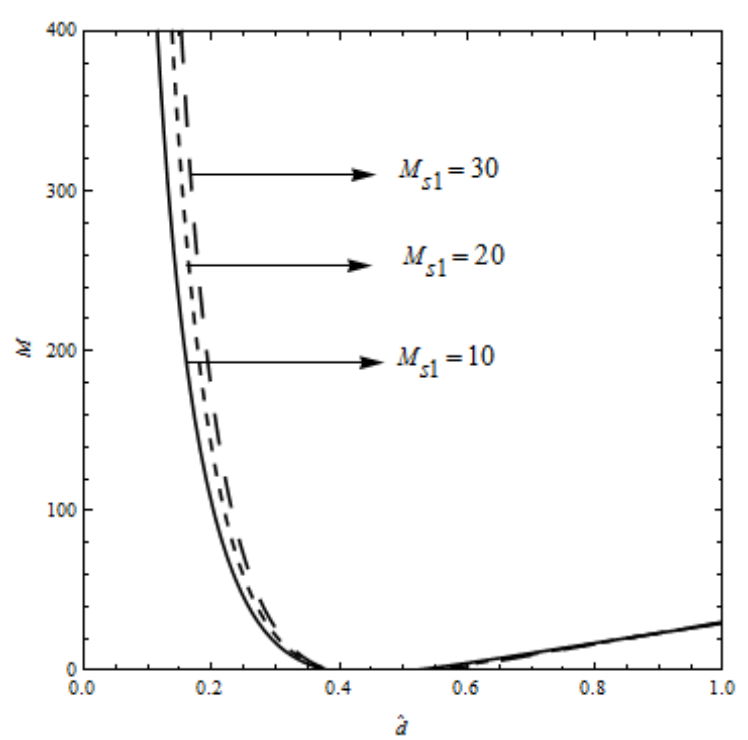

(a)

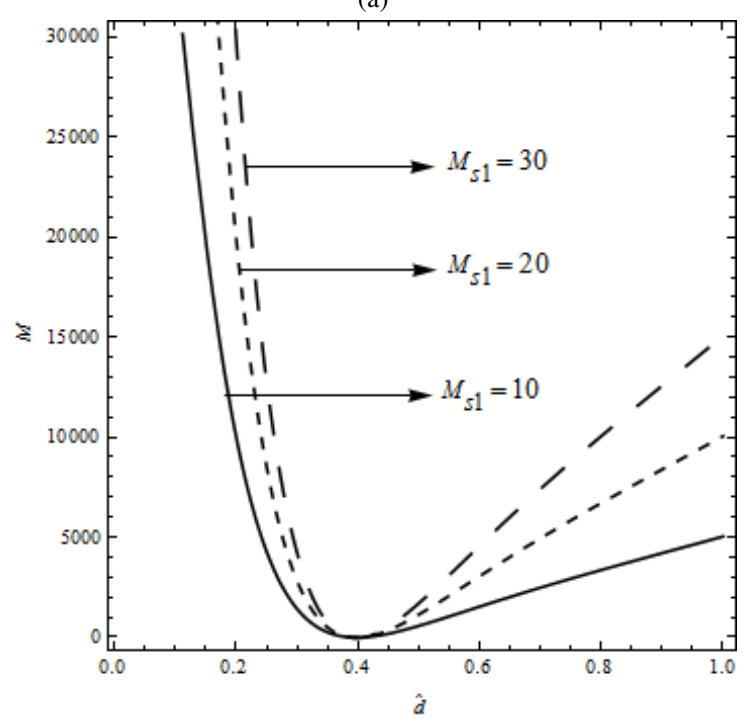

(b)

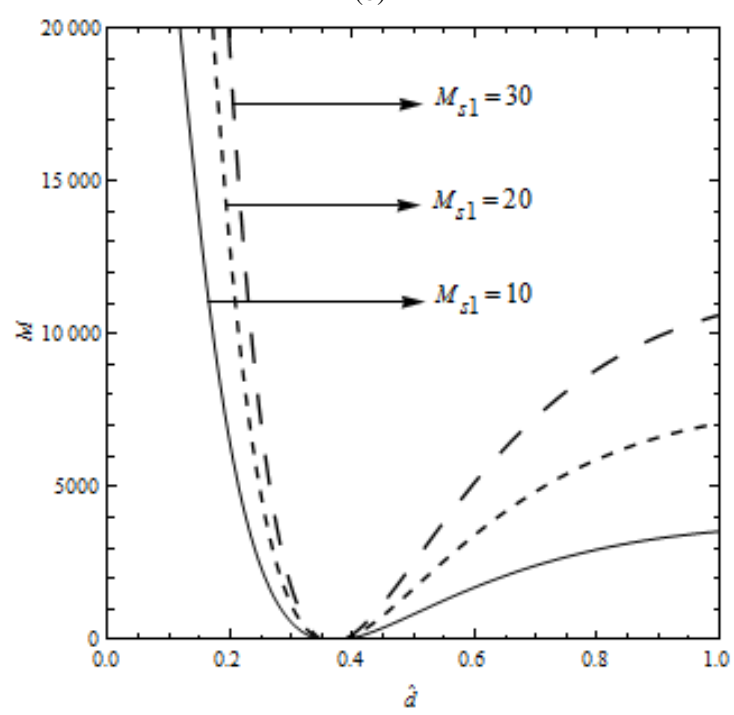

(c)

Figure 7. The effects of solute1 Marangoni number $M_{s 1}$ 
Effects of Non-uniform Temperature Gradients on

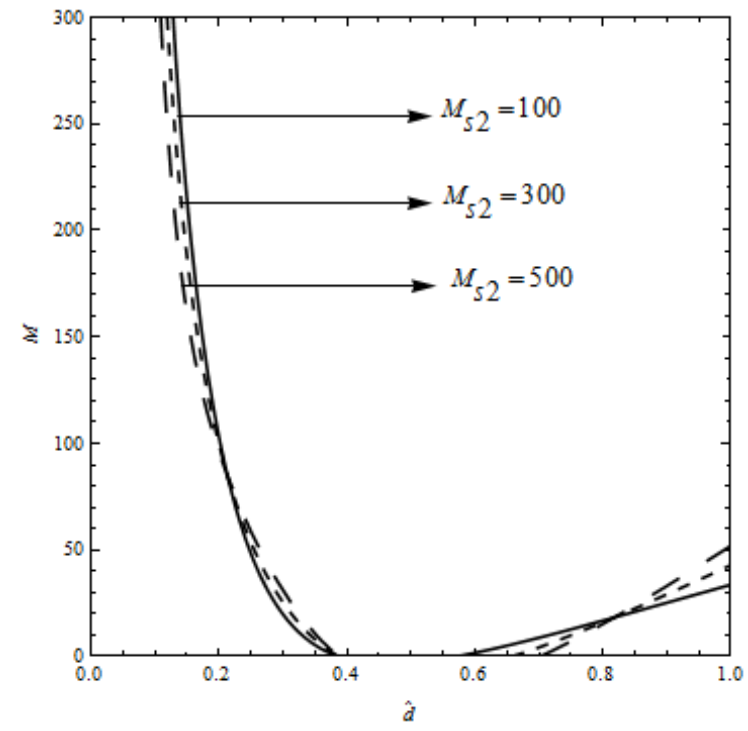

(a)

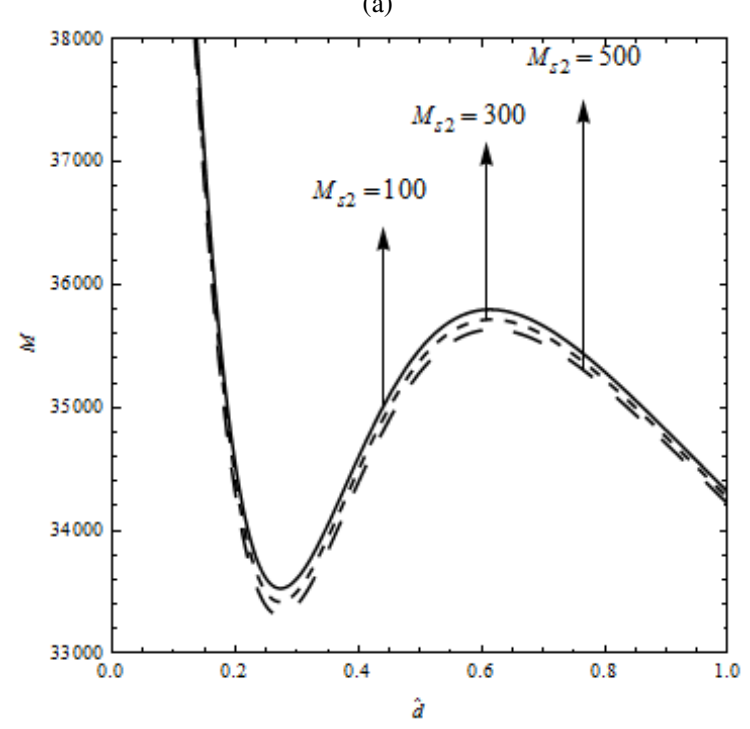

(b)

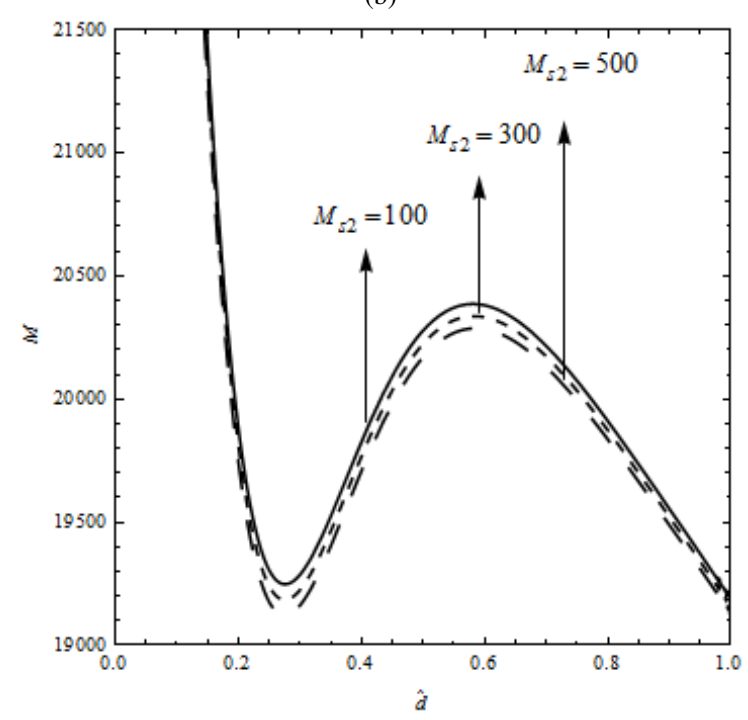

(c)

Figure 8. The effects of solute2 Marangoni number $M_{s 2}$

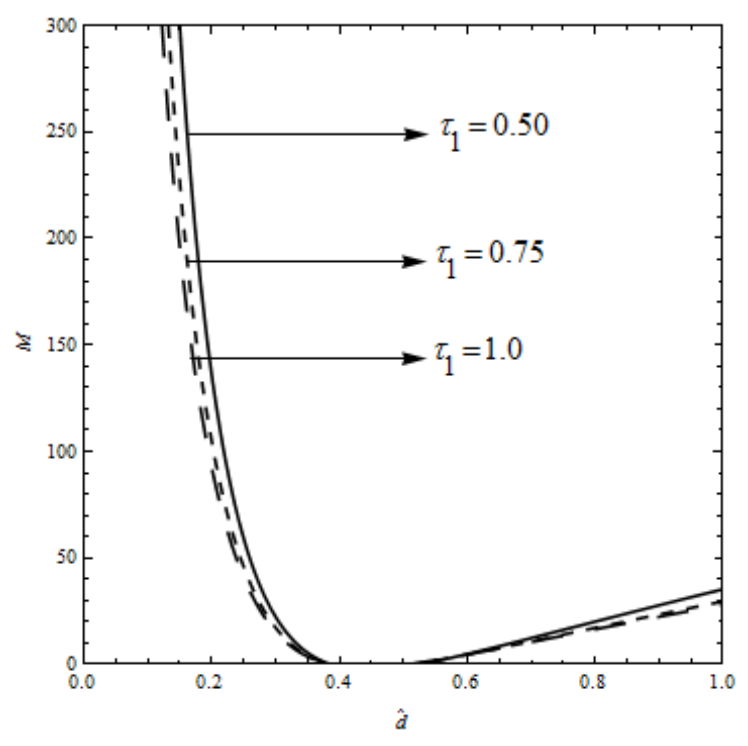

(a)

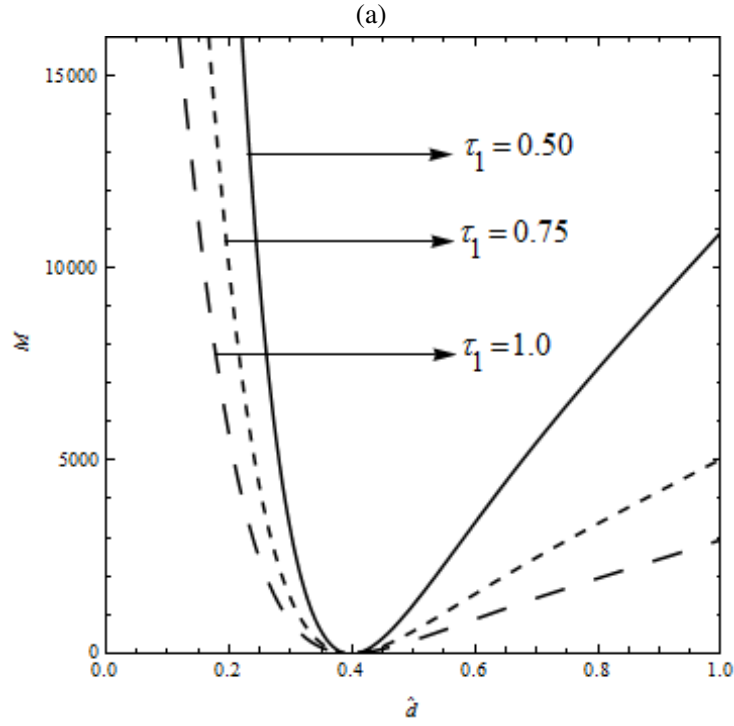

(b)

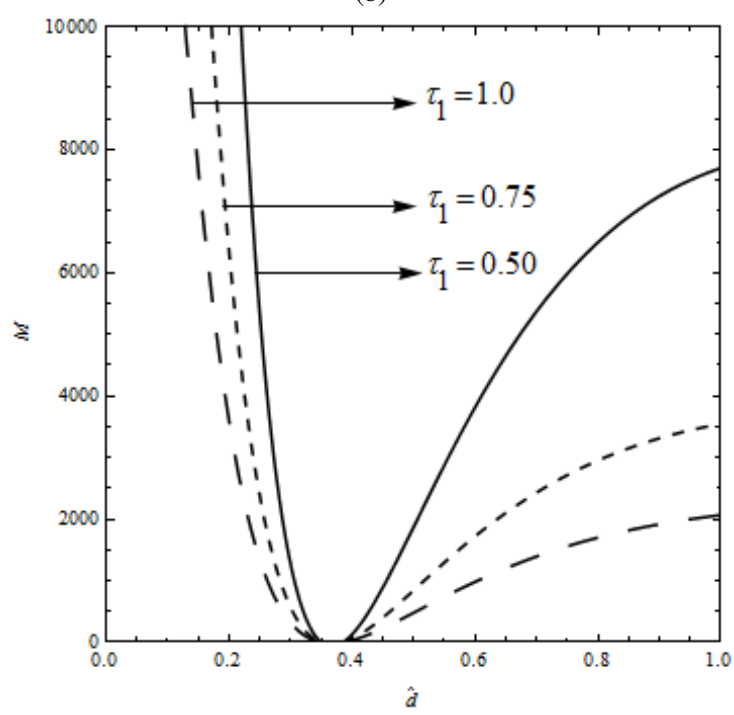

(c)

Figure 9. The effects of $\tau_{1}$ 